\title{
Pinning and de-pinning phenomena in front propagation in heterogeneous media
}

\author{
N. DIRR ${ }^{\dagger}$ \\ Max-Planck Institute for Mathematics in the Sciences, \\ Inselstr. 22, D-04103 Leipzig, Germany \\ AND \\ N. K. YIP \\ Department of Mathematics, Purdue University, \\ West Lafayette, IN 47907, USA
}

[Received 12 May 2005 and in revised form 27 September 2005]

\begin{abstract}
This paper investigates the pinning and de-pinning phenomena of some evolutionary partial differential equations which arise in the modelling of the propagation of phase boundaries in materials under the combined effects of an external driving force $F$ and an underlying heterogeneous environment. The phenomenology is the existence of pinning states-stationary solutions-for small values of $F$, and the appearance of genuine motion when $F$ is above some threshold value. In the case of a periodic medium, we characterise quantitatively, near the transition regime, the scaling behaviour of the interface velocity as a function of $F$. The results are proved for a class of semilinear and reaction-diffusion equations.
\end{abstract}

\section{Introduction}

In this paper we consider the dynamical behaviour of the solutions of two examples of partial differential equations which arise in the modelling of front propagations in a heterogeneous medium. The first example is a semilinear parabolic equation. Let $u(x, t): \mathbb{R}^{n} \times \mathbb{R}_{+} \rightarrow \mathbb{R}$ solve

$$
u_{t}=\Delta u+f(x, u)+F \quad \text { where } u \text { is } \mathbb{Z}^{N} \text {-periodic in } x \text { for all } t \geqslant 0 \text {. }
$$

Here $f(\cdot, \cdot)$ is 1 -periodic in both variables, and sufficiently regular, but not constant in the variable $u$. The constant $F \geqslant 0$ is called the external driving force.

The second is the Allen-Cahn equation with spatially periodic forcing. Let $v(x, t): \mathbb{R} \times \mathbb{R}_{+} \rightarrow$ $\mathbb{R}$ solve

$$
v_{t}=v_{x x}-W^{\prime}(v)+\delta(g(x)+F), \quad x \in \mathbb{R} .
$$

Here $W(v)$ is a double-well potential, i.e. a function with $W(1)=W(-1)=0$ and $W(v)>0$ for $|v| \neq 1$. It usually takes the form of $W(v)=\left(1-v^{2}\right)^{2}$. The function $g(\cdot)$ is 1 -periodic with mean zero. The constant $\delta$ is taken to be positive and sufficiently small. We will concentrate on solutions $v(x, t)$ which have a front structure in the sense that they connect two "different end states" of (2)

\footnotetext{
†E-mail: ndirr@mis.mpg.de

† Corresponding author. E-mail: yip@math.purdue.edu
} 
as $x \rightarrow \pm \infty$. The end states are stationary solutions close to +1 and -1 . These conditions will be specified later.

The solutions of (1) and (2) we are interested in are stationary solutions on one hand and pulsating waves on the other. The latter are solutions which, up to spatial translations, are space-time periodic - the analogue of travelling waves in the case of constant coefficients. We are particularly interested in the transition between these two types of solutions as $F$ varies (increases). More precisely, we will show that for both of the above equations, generically, there exists an $F_{*}-c r i t i c a l$ forcing-such that for $0 \leqslant F \leqslant F_{*}$, the equations have stationary solutions, while for $F>F_{*}$, they have solutions presenting fronts propagating in space with an average velocity $V_{F}$. We also show that in the case of periodic spatial heterogeneity, the velocity satisfies the following scaling law:

$$
V_{F} \sim\left(F-F_{*}\right)^{1 / 2} \quad \text { for } 0<F-F_{*} \ll 1 .
$$

The above described dynamical behaviour should be contrasted with the situation $f \equiv 0$ or $g \equiv 0$ in which it can be shown (in fact it is obvious for (1)) that there exists for any $F \neq 0$ a solution which moves with constant speed: the linear solution $u(x, t)=U_{0}+F t$ in the case of (1) and the travelling wave $u(x, t)=U(x-c t)$ in the case of (2). So the pinning and de-pinning transition is caused by the oscillatory nature of the spatial heterogeneity.

\subsection{Motivations and applications}

Both equations (1) and (2) are simplified models for the motion of an interface in a material under the combined effect of the spatially heterogeneous environment, given by $f(x, u)$ or $g(x)$, the surface tension and the external driving field $F$. The interface could for example be a domain boundary in a magnetic material with impurities [5, Chapter 13], the interface between immiscible fluids in a porous medium [23], or a dislocation line in a solid [16, 17]. The impurities can in general impede or accelerate the motion of the interface, but they are soft obstacles in the sense that the interface can pass through them. The motions of the interface are characterised by the interaction between an interfacial energy, which keeps the interface flat or straight, and the influence of the impurities, which force the interface to deviate from a flat shape, and the driving field, which makes the interface move one way or the other. For an introduction from the physical point of view, we refer to [11] or [17]. Note that in many of the above applications, the driving forces derived from the interfacial energy, the spatial heterogeneity and the external field can all take the form of nonlocal operators.

Another important ingredient in the modelling is the consideration of random impurities. For an overview of models which are characterised by the competition between a surface energy and a random field, we refer to [21]. For rigorous mathematical results on a one-dimensional model, see [14].

In the dynamic case, the motion of an interface in such a model decomposes into periods of slow motion and sudden jerks, called avalanches [11]. In the specific case of (1) with random spatial forcing $f(x, u)$, the model is known in the physics literature as the quenched Edwards-Wilkinson model which is studied extensively (see for example [4]).

While we see the case of periodic impurities treated in this paper mainly as a first step towards a rigorous mathematical analysis of such models for random media, we would like to point out that the periodicity is fully justified from a modelling point of view if the impurity is related to a periodic crystal structure of an underlying substrate.

Already in the periodic case, the relationship between the mean velocity and the driving force is expected to be a power law near the transition regime, which is due to the interplay between some 
slow motion near the stationary solutions at $F=F_{*}$ and fast motion with speed of order one away from the stationary states.

In order to keep the mathematical treatment simple, we consider spatially localised driving forces. As the mean curvature is the first variation of the length or area of the interface which is a simple form of the interfacial energy, one way to model the above phenomenology is by means of the mean curvature flow with a driving force: the normal velocity $V_{n}$ at any point on the interface $\Gamma(t)$ is given by the following motion law:

$$
V_{n}=\kappa-f(x)+F \quad \text { for } x \in \Gamma(t) .
$$

Here $\kappa$ is the mean curvature of the interface, $f(x)$ is some spatial function and $F$ is a constant.

Even in this case, the geometry of the interface can become quite complicated because selfintersection can occur. In addition, the graph representation of the interface might not be preserved under the dynamics. One reason equations (1) and (2) are used is to avoid this technical difficulty. For (1), a linearised version of the curvature operator $\kappa$ is used and the interface is given by the graph of the function $u(x, t)$. In this way, the graph representation is preserved for all time and estimates of the solutions are easier to obtain.

The second equation (2) (when $\delta=0$ ) is a gradient flow of a Ginzburg-Landau type free energy for a physical system with a nonconserved order parameter and two stable phases corresponding to the two "wells" \pm 1 of $W$ [2]. It is well known that under large space-time diffusive rescaling, i.e. $t \rightarrow \epsilon^{2} t$ and $x \rightarrow \epsilon x$, the zero level set of suitable solutions converges to an interface moving by its mean curvature [22]. Thus equation (2) is an approximation of (4) on some large spatial and temporal scale, while it is a model in its own right on a smaller scale, usually called the mesoscopic scale.

Of course, in the one-dimensional case studied here the limiting motion is the motion of a point in a spatial potential. Let $g$ be a function of period one. Then under the above mentioned spacetime scaling, the zero $R(t)$ of $v(x, t)$ which represents the front location, evolves according to the following ODE:

$$
\mu \dot{R}=-\Phi^{\prime}(R)
$$

where $\Phi(R)=-R F-G(R)$, and $G(R)$ is a 1-periodic potential such that $G^{\prime}(R)=g(R)$. The constant $\mu$ is usually called the mobility. In essence, equation (5) describes the negative gradient flow of a particle in a tilted periodic potential. This simple ODE already shows some of the qualitative features of the more complex PDEs such as (1) and (2). Thus it is advantageous to briefly describe its behaviour here.

For $F=0$ the function $G(R)$ is assumed to have global minima periodically spaced leading to stable stationary solutions. When $F=F_{*}=-g\left(R_{*}\right)$, where $g\left(R_{*}\right)=\inf _{[0,1]} g(x)$, a stationary solution $R(t) \equiv R_{*}$ still exists. Note that at this point, $g^{\prime}\left(R_{*}\right)=0$. Now let $F=F_{*}+\gamma$, and $y=R-R_{*}$. If we further assume the nondegeneracy condition $\alpha=g^{\prime \prime}\left(R_{*}\right)>0$, then

$$
\dot{y} \sim \alpha y^{2}+\gamma \text { for } y \text { near } 0 \text { and } 0<\gamma \ll 1 \text {. }
$$

The explicit solution is given by $y(t)=(\gamma / \alpha)^{1 / 2} \tan \left((\alpha \gamma)^{1 / 2} t\right)$. Thus it takes the solution a time of order $\gamma^{-1 / 2}$ to leave the region of slow motion and to reach a region of fast motion where the speed becomes independent of $\gamma$.

The above type of argument is also used in [8] in the study of the pinning and de-pinning phenomena for some discrete reaction-diffusion equations. Equation (6) in fact describes the 
dynamics along the centre manifold at the critical point $y=0$. The work [1] also uses an equation similar to (5) in the investigation of the dynamics of some martensitic phase transformations.

\subsection{Statement of theorems}

Here we state precisely the definitions and theorems to be shown in this paper.

For the semilinear equation [1], we first introduce the linearised operator $L_{f}(u)$ at a function $u(x)$ :

$$
L_{f}(u) \varphi:=\Delta \varphi+\partial_{u} f(x, u(x)) \varphi \quad \text { for any } \mathbb{Z}^{n} \text {-periodic } \varphi .
$$

The function $f(x, u): \mathbb{R} \times \mathbb{R} \rightarrow \mathbb{R}$ satisfies the following conditions:

A1 $\bullet$ is $\mathbb{Z}^{n}$-periodic in $x$ and and 1-periodic in $u$, i.e.

$$
f(x, u+1)=f(x, u) \text { and } f(x+\hat{e}, u)=f(x, u) \quad \text { for all }(x, u) \in \mathbb{R}^{n} \times \mathbb{R},
$$

where $\hat{e}$ is the unit vector along any coordinate axis.

- $f(x, u)$ is twice continuously differentiable in both variables and $\|f(\cdot, \cdot)\|_{C^{2}\left(\mathbb{R}^{n} \times \mathbb{R}\right)}<\infty$.

A2 At $F=0$, equation (1) has a stationary periodic solution $u_{0}(x)$ which is stable in the sense that the principal eigenvalue of the linearised operator $L_{f}\left(u_{0}\right)$ is negative.

Note that the mere existence of a stationary solution at $F=0$ is nothing more than a normalisation and follows e.g. if the average of $f(x, u)$ is zero (see Lemma 4). The stability condition is indeed an assumption and excludes for example any $f(x, u)$ which is constant in the variable $u$. The regularity assumption on the nonlinearity serves to keep the technical effort at a minimum. We expect that it can be relaxed.

Theorem 1 Assume A1 and A2. Then the following statements hold.

1. There exists an $F_{*}>0$ such that for $0 \leqslant F \leqslant F_{*}$, (1) has a stationary solution $U_{F}(x)$.

2. Assume in addition the condition $\mathbf{A 3}$ (given right afterward), or that there exist only finitely many stationary solutions (up to translation) at $F=F_{*}$. Then, for $F>F_{*}$, there exists a unique $0<T_{F}<\infty$ and a solution $U_{F}(x, t)$ of (1) satisfying

$$
U_{F}(x+\hat{e}, \cdot)=U_{F}(x, \cdot) \quad \text { and } \quad U_{F}\left(\cdot, t+T_{F}\right)=U_{F}(\cdot, t)+1 .
$$

The condition $\mathbf{A} \mathbf{3}$ is as follows:

A3 For any stationary solution $u_{*}$ at $F_{*}$ we have

$$
\int_{Q} \varphi_{p}^{3} \partial_{u}^{2} f\left(x, u_{*}(x)\right) \mathrm{d} x \neq 0,
$$

where $Q=[0,1]^{n}$ and $\varphi_{p}$ is the principal eigenfunction of $L_{f}\left(u_{*}\right)$.

The above is reminiscent of some nondegeneracy condition. It corresponds to the fact that $g^{\prime \prime}\left(R_{*}\right) \neq$ 0 for the ODE example (5). (In fact, this assumption implies that the above quantity is automatically positive. See Claim I on page 90, In addition, it is also used to give the precise asymptotics of the dynamics for $F>F_{*}$. Otherwise, some higher (for example, fourth) order information is needed.

Now we are ready to present the asymptotics of the propagating velocity: 
Theorem 2 Assume A1, A2 and A3. Then there is a constant $A$ (see (39) for its formula) such that the average velocity $V_{F}$ defined as $T_{F}^{-1}$ has the following asymptotics:

$$
V_{F}=A\left(F-F_{*}\right)^{1 / 2}+o\left(\left|F-F_{*}\right|^{1 / 2}\right) \quad \text { for } 0<F-F_{*} \ll 1 .
$$

REMARK. Note that even though we only consider solutions which are periodic in the spatial variable, this is not as restrictive as it seems because the comparison principle implies that the periodic solutions can already determine the pinning and de-pinning behaviour of arbitrary solutions which are bounded in the $L^{\infty}$ norm.

In addition, in Section 2.4, we can actually show that there are lots of functions $f(x, u)$ satisfying A1-A3. See also Section 4.1 for an explicit example.

For the inhomogeneous Allen-Cahn equation (2), we consider the regime of weak spatial heterogeneity. In this case, it turns out that we can obtain quite explicit quantitative information on the speed of the pulsating wave. First we introduce the standing wave solution $m(x)$ (for $\delta=0)$ :

$$
m_{x x}-W^{\prime}(m)=0
$$

such that for some constant $C$ it satisfies the estimates

$$
|| m(x)|-1| \leqslant C e^{-a|x|} \text { and }\left|m_{x}(x)\right| \leqslant C e^{-a|x|} \quad \text { for all }-\infty<x<\infty
$$

where $a$ is any positive number less than $W^{\prime \prime}( \pm 1)$. (The existence of $m(x)$ and its properties are well known [10].) We next introduce the 1-periodic function

$$
h(a)=-\int_{-\infty}^{\infty} g(z+a) m_{z}(z) \mathrm{d} z .
$$

Without loss of generality, assume that the minimum value of $h$, denoted by $h_{*}$, is attained at $a=$ $0, \pm 1, \pm 2, \ldots$ Assume further that $h^{\prime \prime}(0)>0$. We first define the following quantities:

$$
F_{*}=-\frac{h_{*}}{2}, \quad \alpha=\frac{2}{\left\|m_{x}\right\|^{2}}, \quad \beta=\frac{h^{\prime \prime}(0)}{4\left\|m_{x}\right\|^{2}}
$$

where $\left\|m_{x}\right\|^{2}=\int_{-\infty}^{\infty} m_{x}(x)^{2} \mathrm{~d} x$. Then we have:

Theorem 3 For any $\sigma>0$, there exist $\delta_{*}, C_{1}, C_{2}>0$ such that for any $\delta<\delta_{*}$ and any $F$ in the range

$$
0<C_{1} \delta \leqslant F-F_{*} \leqslant C_{2}
$$

there exists a constant $T_{F}$ and a solution $U_{F}(x, t)$ of (2) satisfying

$$
U_{F}\left(x, t+T_{F}\right)=U_{F}(x-1, t) .
$$

In addition, the velocity of the front, defined as $V_{F}=T_{F}^{-1}$, satisfies

$$
(1-\sigma) \frac{\alpha \delta \sqrt{\beta\left(F-F_{*}\right)}}{\pi} \leqslant V_{F} \leqslant(1+\sigma) \frac{\alpha \delta \sqrt{\beta\left(F-F_{*}\right)}}{\pi} .
$$




\subsection{Structure of the paper and outline of proofs}

The dynamics of (1) and (2) is analysed in Sections 2 and 3 respectively. In Section 4, we provide some explicit examples of the pinning phenomena. These examples are illustrative in the sense that they clearly demonstrate the existence of a nontrivial pinning state. Furthermore, the constants in the abstract formulation can be computed analytically. In Appendix B, for the reader's convenience, the spectral estimates for the linearised Allen-Cahn operator are provided.

Here we briefly describe the method of the proof. For the semilinear case, we first show the existence of a threshold force and some properties of the stationary solutions. In particular, we prove that the threshold value $F_{*}$ is characterised by the fact that the principal eigenvalue of the linearised operator at any stationary solution must be zero. The principal eigenfunction and the nondegeneracy condition A3 are then used to construct sub- and supersolutions near the critical solution. In this way, we can deduce the time a solution spends near a pinned state for $F$ slightly larger than $F_{*}$. This is reminiscent of the analysis of the dynamics on the centre-manifold of a stationary solution. Sharp asymptotic estimates for the period of the pulsating wave are also available. The overall phenomenology is very similar to the behaviour already revealed in the simple ODE model (5) and its analysis (6). The existence of pulsating wave solutions is then obtained by the Schauder fixed point theorem. The contracting property of the heat semigroup is crucial in setting up the nonlinear map used in the fixed point theorem.

In Section 3, we obtain the existence of pulsating wave solutions for (2) and quantitative information about their speed of propagation. We work in the weakly spatial heterogeneity regime $(0<\delta \ll 1)$ so that we can employ the techniques of perturbation theory. We heavily use the spectral properties of a one-parameter manifold of stationary solutions of the unperturbed equation $(\delta=0)$. Even though it is conceivable that a proof similar to the one in the semilinear case is also available for the reaction-diffusion case so that $\delta$ might not be constrained to be small, we believe that the strategy employed here provides a different perspective and most importantly, it gives explicit quantitative information about the threshold value $F_{*}$ and the speed of the propagating front.

Here we also mention some related results. The work [20] studies similar equations to (1) but their spatial forcing is required to be positive everywhere. In the sequence of works [26, 27, 28], the pinning phenomena is investigated for the spatially forced Allen-Cahn equation (2). This is commonly referred to as the bi-stable case. The existence of pulsating waves is also proved. The inhomogeneity in [26, 27, 28] is different from the case studied in this paper. Recently, [6, 7], and, within a more general framework, [18], analyse similar front propagating phenomena and establish some homogenisation results in the setting of Hamilton-Jacobi and degenerate parabolic differential equations. However, in all of these works, the pinning and de-pinning transition is not studied which is the main emphasis here.

\section{The semilinear equation: Proofs}

In this section we prove Theorems 1 and 2 for the semilinear equation (1). They are consequences of several propositions.

As the analysis of the dynamics relies very much on the properties of the stationary solutions, we state here the static version of equation (1):

$$
0=\Delta u+f(x, u)+F, \quad \text { where } u \text { is } \mathbb{Z}^{n} \text {-periodic in } x \in \mathbb{R}^{n} .
$$


Recall that $F$ is a constant and that $f(\cdot, \cdot)$ fulfills A1. An important role will be played by the linearised operator (7) at a function $u(x)$, which is stated here again for convenience:

$$
L_{f}(u) \varphi=\Delta \varphi+\partial_{u} f(x, u) \varphi, \quad \text { where } \varphi \in H^{1}\left(\mathbb{R}^{n}\right) \text { is } \mathbb{Z}^{n} \text {-periodic. }
$$

The eigenvalue problem for $L_{f}(u)$ is given by

$$
L_{f}(u) \varphi=\lambda \varphi \quad \text { for } \varphi \in H^{1}\left(\mathbb{R}^{n}\right) \text { and } \mathbb{Z}^{n} \text {-periodic. }
$$

First we show a proposition which makes assumption A2 more natural.

Proposition 4 If $f(x, u)$ satisfies $\mathbf{A 1}$ and

$$
\int_{0}^{1} \int_{Q} f(u, x) \mathrm{d} x \mathrm{~d} u=0
$$

then equation (14) has a solution for $F=0$.

Proof. Note that the energy functional

$$
E(u):=\frac{1}{2} \int_{Q}|\nabla u|^{2} \mathrm{~d} x-\int_{Q} H(u, x) \mathrm{d} x, \quad \text { where } H(u, x)=\int_{0}^{u} f(u, x) \mathrm{d} u,
$$

is lower semicontinuous if $u_{n} \rightarrow u$ strongly and $\nabla u_{n} \rightarrow \nabla u$ weakly in $L^{2}$. In addition, because of

$$
\left|\int H(x, u(x)) \mathrm{d} x\right| \leqslant C\|u\|_{L^{1}},
$$

the energy is bounded from below. Thus $E(u)$ has a minimiser in the class $H_{s}^{1}$ of all periodic $H^{1}$-functions with $\int_{Q} u(x) \mathrm{d} x=s$.

Let $G(s):=\min _{H_{s}^{1}} E(u)$. Note that as $f$ has zero average, we have $\int_{Q} H(u+1, x) \mathrm{d} x=$ $\int_{Q} H(u, x) \mathrm{d} x$. Hence $G(s+1)=G(s)$. Comparing $E(u)$ and $E(u+\epsilon)$, we see that $G$ is Lipschitz, so $G$ assumes its minimum for some $s_{0}$. This minimum is attained at some function $u_{0}$. Therefore a minimiser of $E$ in the class of periodic $H^{1}$ functions exists. This function $u_{0}$ solves the EulerLagrange equation associated with $E$, which is 114 with $F=0$.

REMARK. The $u_{0}$ from the above proof is stable in the sense of minimisation. It can further be made stable in the sense of $\mathbf{A} \mathbf{2}$ upon changing $f$ to

$$
f(x, u)+\epsilon R\left(u-u_{0}(x)\right)-\epsilon \int_{0}^{1} \int_{Q} R\left(u-u_{0}(x)\right) \mathrm{d} x \mathrm{~d} u
$$

(and extended periodically in $x$ and $u$ ), where $0<\epsilon \ll 1$ and $R$ is a smooth function such that $R(s)=-s$ for $|s| \leqslant 1 / 8$ and $R(s)=0$ for $|s| \geqslant 1 / 4$.

The proof of Theorem 1 is divided into several propositions. For convenience, we use the notation $(1)_{F}$ and $(14)_{F}$ to denote the dependence of the equations on $F$. 
Proposition 5 (Existence of threshold force) Assume A1 and A2.

1. There exists an $F_{*}>0$ such that $(14)_{F}$ has a classical solution for $0 \leqslant F \leqslant F_{*}$ while it has no solution for $F>F_{*}$.

2. At $F=F_{*}$, the collection of the stationary solutions is well ordered in the sense that if $u_{1}$ and $u_{2}$ are two solutions of $(14)_{F_{*}}$, then either $u_{1}<u_{2}$ or $u_{2}<u_{1}$.

3. Let $u$ be a solution of $(14)_{F_{*}}$. Then the principal eigenvalue of the linearised operator $L_{f}(u)$ (7) is zero and simple and the corresponding eigenvector $\varphi_{p}(\cdot)$ is strictly positive.

Proof of 5[1]). Consider $\mathcal{F}=\left\{F \geqslant 0\right.$ : equation $(14)_{F}$ has a solution $\}$. By assumption, $0 \in \mathcal{F}$ $\neq \emptyset$. On the other hand, if $F>\|f\|_{\infty}$, then $[14)_{F}$ has no solution. Hence we can define $F_{*}=$ $\sup \{F \in \mathcal{F}\}<\infty$. We claim that $F_{*} \in \mathcal{F}$. For if not, there exists a sequence of $F_{n}$ converging to $F_{*}$ and also a sequence of functions $u_{n}(x)$ satisfying $\left(14{ }_{F_{n}}: \Delta u_{n}+f\left(x, u_{n}\right)+F_{n}=0\right.$. Since $f$ satisfies $\mathbf{A} 1$ and the $F_{n}$ 's are uniformly bounded, by elliptic regularity, the collection of $\left\{u_{n}\right\}$ 's is compact and hence has a subsequence converging to some $u_{*}$ satisfying $\Delta u_{*}+f\left(x, u_{*}\right)+F_{*}=0$. Thus $F_{*} \in \mathcal{F}$.

According to $\mathbf{A 2}$, the linearised operator $L_{f}\left(u_{0}\right)$ at a solution $u_{0}$ of $14 p_{0}$ has negative principal eigenvalue. Thus $L_{f}\left(u_{0}\right)$ is invertible. A straightforward application of the implicit function theorem (see for example [3]) implies that $[14)_{F}$ also has a solution for any $F$ which is slightly larger than 0. Hence $F_{*}>0$.

Next we show that $(14)_{F}$ has a solution for any $0<F<F_{*}$. Consider the solution $u_{*}$ of $\left(14 F_{*}\right.$ and $u_{0}$ of $(14)_{0}$. Then they are also super- and subsolutions of $(14)_{F}$, respectively. By the periodicity of $f(x, u)$ in the $u$ variable, we can assume that $u_{0}<u_{*}+N$ for some large enough integer $N$. Hence $(14)_{F}$ must have a solution for any $F$ in $\left(0, F_{*}\right)$.

Proof of 5[2]. Let $u_{1}$ and $u_{2}$ be two solutions of $(14)_{F_{*}}$. Suppose the graphs corresponding to $u_{1}$ and $u_{2}$ cross each other. Consider

$$
U(x)=\min \left\{u_{1}(x), u_{2}(x)\right\} \quad \text { and } \quad V(x)=\max \left\{u_{1}(x), u_{2}(x)\right\} .
$$

Now $U(x)$ and $V(x)$ are sub- and supersolutions of $\left[14 F_{*}\right.$, but not solutions. Again, by translating $U$ or $V$ in the $u$-direction, we can assume without loss of generality that $V<U$. The idea next is to show that they are still super- and subsolutions for $(1)_{*}+\gamma$ for some small enough and positive $\gamma$. Thus a stationary solution for $1 F_{F_{*}+\gamma}$ exists, contradicting the definition of $F_{*}$.

This is made precise as follows. Consider the solution $u(x, t)$ of the evolution equation (1) $F_{*}$ with initial data $U(x)$. Then $u(x, h)<u(x, 0)$ for any $h>0$. The regularity assumptions satisfied by $f(x, u)$ imply that for $0<h \ll 1$ the function $v_{h}(x, t):=u(x, t)-u(x, t+h)$ solves the linear equation

$$
\left(v_{h}\right)_{t}=\Delta v_{h}+c(x, t) v_{h}, \quad v(x, 0) \geqslant 0,
$$

with a uniformly bounded potential $c(x, t)$. As a consequence, $v_{h}(x, t)$ cannot attain a negative minimum, which leads to $u(x, t+h) \leqslant u(x, t)$ and hence $u_{t}(x, t) \leqslant 0$ but $\not \equiv 0$ for $t>0$. Differentiating (1) $F_{*}$ with respect to $t$ shows that the function $u_{t}$ solves a linear parabolic differential equation with bounded coefficients. By the strong maximum principle, there exists a $t_{1}>0$ and $c$ such that $u_{t}\left(\cdot, t_{1}\right)<-c<0$. Now consider $(1) F_{*}+\gamma$ for $0<\gamma<c$ and the initial data $u\left(x, t_{1}\right)$. Its solution $u_{2}(x, t)$ will decrease monotonically in time. On the other hand, using $V(x)$ we can construct in a similar way a solution $u_{3}(x, t)$ of $\sqrt{1} F_{*}+\gamma$ which will increase monotonically in time. Hence there must be a stationary solution of $(14)_{F_{*}+\gamma}$ leading to the desired contradiction. 
Proof of 5 3). Let $u_{*}$ be a solution of $(14)_{F_{*}}$. By the spectral theorem for compact self-adjoint operators, the spectrum of $L_{f}\left(u_{*}\right)$ consists of discrete eigenvalues: $\lambda_{p} \geqslant \lambda_{1} \geqslant \cdots \rightarrow-\infty$. The variational characterisation of $\lambda_{p}$, using the Rayleigh quotient, implies that $\lambda_{p}$ is simple, i.e. of multiplicity one, and its eigenfunction $\varphi_{p}$ is nonnegative. The fact that $\varphi_{p}$ is strictly positive follows from the strong maximum principle. Now suppose $\lambda_{p} \neq 0$. Again, the idea is to construct sub- and supersolutions of $(14)_{*}+\gamma$ for some small but positive $\gamma$. Let $0<\epsilon \ll 1$. Consider the function

$$
v_{\epsilon}^{ \pm}(x, t)=u(x, t) \pm \epsilon \varphi_{p}(x) .
$$

By linearising the nonlinearity $f(u, x)$ up to second order, we obtain

$$
\Delta\left(v_{\epsilon}^{ \pm}\right)+f\left(\cdot, v_{\epsilon}^{ \pm}\right)+F_{*}+\gamma= \pm \epsilon\left(\lambda_{p} \varphi_{p}+O(\epsilon)\right)+\gamma
$$

Assume $\lambda_{p}>0$. As $\varphi_{p}(x)$ is strictly positive, we can first choose an $\epsilon$ and then a $\gamma>0$ such that $v_{\epsilon}^{+}$is a supersolution of $14 F_{*}+\gamma$, whereas $v_{\epsilon}^{-}$is a subsolution. Hence, by reasoning as before, we see that there must be a stationary solution for $F^{*}+\gamma$ between $v_{\epsilon}^{+}$and $v_{\epsilon}^{-}+N$ for some large integer $N$, which again contradicts the definition of $F^{*}$. The case of $\lambda_{p}<0$ follows similarly.

Proposition 6 (Uniqueness of space-time periodic solution) Let $F_{*}$ be as in Proposition 5 . Then for any $F>F_{*}$, if a space-time periodic solution satisfying (8) exists, then the time period $T_{F}$ is unique. In addition, the solution is unique up to a time shift, i.e. if $U_{F}^{1}, U_{F}^{2}$ are two such solutions, then for some $t_{0}$,

$$
U_{F}^{1}(x, t)=U_{F}^{2}\left(x, t-t_{0}\right)
$$

Proof. Let $U_{1}(x, t)$ and $U_{2}(x, t)$ be two space-time periodic solutions of $(1)_{F}$ with time-periods $\infty>T_{1}>T_{2}>0$. Now consider solutions $u_{1}(x, t)$ and $u_{2}(x, t)$ of $(1)_{F}$ with initial data $U_{1}(x, 0)+$ $N$ and $U_{2}(x, 0)$ where $N$ is a large enough positive integer such that $U_{1}(x, 0)+N>U_{2}(x, 0)$. By (8), there must be a $t_{1}$ and $x_{1}$ such that $u_{2}\left(x_{1}, t_{1}\right)=u_{1}\left(x_{1}, t_{1}\right)$, contradicting the comparison principle. Thus $T_{1}=T_{2}=T_{F}$

Now consider again the solution $U_{2}(x, t)$. There must be a time $t_{2}$ and $x_{2}$ such that $U_{2}\left(x_{2}, t_{2}\right)=$ $U_{1}\left(x_{2}, 0\right)+N$ and $U_{2}\left(\cdot, t_{2}\right) \leqslant U_{1}(\cdot, 0)+N$ for some large positive integer $N$. Now solve (1) $F$ with initial data $U_{1}(x, 0)+N$ and $U_{2}\left(x, t_{2}\right)$. By time periodicity, after a time $T$, the two solutions will touch again-contradicting the comparison principle, unless $U_{1} \equiv U_{2}$ (up to an additive integer). Thus the space-time periodic solution must be unique in the sense stated in the proposition.

Proposition 7 (Existence of space-time periodic solution) For each $F>F_{*}$, there exists a number $0<T_{F}<\infty$ and a function $U_{F}(x, t)$ satisfying $(1) F$ and $(8)$.

Proof. It is easy to show that under assumption A1, the initial value problem $(1)_{F}$ is well-posed in $L^{2}$ and a solution exists globally in time. We will employ the Schauder fixed point theorem to prove the existence of a solution satisfying $(8)$.

Let $u(x, t)$ be a solution of $11 F$. It can be decomposed as $u(x, t)=p(t)+\xi(x, t)$ with $\langle\xi(\cdot, t)\rangle=0$, where $\langle g(\cdot)\rangle=\int_{Q} g(x) \mathrm{d} x$. Then $p(t)$ and $\xi(\cdot, t)$ satisfy

$$
\left\{\begin{array}{l}
\dot{p}(t)=\langle f(\cdot, p(t)+\xi(\cdot))+F\rangle \\
\xi_{t}(x, t)-\Delta \xi(x, t)=f(x, p(t)+\xi(x, t))-\langle f(\cdot, p(t)+\xi(\cdot, t))\rangle \\
\langle\xi(\cdot, t)\rangle=0
\end{array}\right.
$$


Let $\xi_{0}(\cdot)=\xi(\cdot, 0)$ and assume as a normalisation condition that $p(0)=\left\langle u_{*}(x)\right\rangle$. Furthermore, let $\left\|\xi_{0}\right\|_{L^{\infty}}<A$ for some constant $A$. We claim that for any $F>F_{*}$, there exists a unique time $T=T\left(\xi_{0}\right)$ such that $p\left(T\left(\xi_{0}\right)\right)=p(0)+1$ and constants $0<\tau_{1}<\tau_{2}$ independent of $\xi_{0}$ such that

$$
0<\tau_{1}<T\left(\xi_{0}\right)<\tau_{2}<\infty
$$

An obvious lower bound for $T\left(\xi_{0}\right)$ can of course be obtained from $|\dot{p}| \leqslant\|f\|_{L^{\infty}}$ so that $\|f\|_{L^{\infty}}^{-1}<$ $T\left(\xi_{0}\right)$. But an upper bound follows from the centre manifold type analysis as described in Theorem 11 (which also gives much more precise estimates).

Now let $S(t)$ be the solution operator for the heat equation $v_{t}=\Delta v$ on $Q=[0,1]^{n}$ with periodic boundary condition. Let also $\psi(x, t)=f(x, p(t)+\xi(x, t))-\langle f(\cdot, p(t)+\xi(\cdot, t))\rangle$. We introduce the nonlinear map

$$
\mathcal{T}\left(\xi_{0}\right)=\xi\left(x, T\left(\xi_{0}\right)\right) \quad \text { which equals } \quad S\left(T\left(\xi_{0}\right)\right) \xi_{0}+\int_{0}^{T\left(\xi_{0}\right)} S\left(T\left(\xi_{0}\right)-s\right) \psi(\cdot, s) \mathrm{d} s .
$$

The existence of a space-time periodic solution is equivalent to finding a $\xi_{0}(\cdot)$ such that $\xi_{0}=\mathcal{T}\left(\xi_{0}\right)$.

To analyse the map $\mathcal{T}$, we introduce the closed subset of $L^{2}(Q)$,

$$
\mathcal{B}_{A}=\left\{v:\|v\|_{L^{\infty}} \leqslant A,\langle v\rangle=0\right\}
$$

where $A$ is to be determined. First note that $\mathcal{T}$ is a continuous function on $\mathcal{B}$. This follows from the continuous dependence of $\xi(\cdot, t)$ on $t$ and $T\left(\xi_{0}\right)$ on $\xi_{0}$. Note that we only need to consider those times $t$ satisfying (18). Next, we claim the following property for $S(t)$ (which is proved immediately afterwards):

For each $\tau>0$, there exists a $0<C_{1}(\tau)<1$ such that for all $v(\cdot)$, 1-periodic with $\langle v\rangle=0$,

$$
\|S(\tau) v\|_{L^{\infty}} \leqslant C_{1}(\tau)\|v\|_{L^{\infty}}
$$

and there are constants $M$ and $N$ such that

$$
\|S(t) v\|_{L^{\infty}} \leqslant M e^{-N t}\|v\|_{L^{\infty}} \quad \text { for } t>0 .
$$

With the above estimates and the lower bound for $T=T\left(\xi_{0}\right)$, we have

$$
\begin{aligned}
& \left\|\mathcal{T}\left(\xi_{0}\right)\right\|_{L^{\infty}} \leqslant\left\|S(T) \xi_{0}\right\|_{L^{\infty}}+\int_{0}^{T}\|S(T-s) \psi(x, s)\|_{L^{\infty}} \mathrm{d} s \\
& \leqslant C_{1}\left(\tau_{1}\right)\left\|\xi_{0}\right\|_{L^{\infty}}+\int_{0}^{T-1}\|S(T-s) \psi(x, s)\|_{L^{\infty}} \mathrm{d} s+\int_{T-1}^{T}\|S(T-s) \psi(x, s)\|_{L^{\infty}} \mathrm{d} s \\
& \leqslant C_{1}\left(\tau_{1}\right)\left\|\xi_{0}\right\|_{L^{\infty}}+\int_{0}^{T-1} M e^{-N(T-s)}\|\psi(\cdot, s)\|_{L^{\infty}} \mathrm{d} s+C_{2} \\
& \leqslant C_{1}\left(\tau_{1}\right)\left\|\xi_{0}\right\|_{L^{\infty}}+C_{3}
\end{aligned}
$$

for some $0<C_{3}<\infty$. In the above, we have used the fact that $\|\psi(\cdot, s)\|_{L^{\infty}} \leqslant 2\|f\|_{L^{\infty}}<\infty$.

Now let $A=C_{3} /\left(1-C_{1}\left(\tau_{1}\right)\right)$. Then $\left\|\xi_{0}\right\| \leqslant A$ implies that $\|\mathcal{T}(\xi)\|_{L^{\infty}} \leqslant A$. Thus $\mathcal{T}(\cdot)$ maps $\mathcal{B}_{A}$ into itself. The lower bound for $T$ together with parabolic regularity leads to the compactness of 
the map $\mathcal{T}$ in $L^{2}$. Hence the Schauder fixed point theorem (see [13, Corollary 11.2]) implies that $\mathcal{T}$ has a fixed point.

Proof of (20). First, by maximum principle, $\|S(t) v\|_{L^{\infty}} \leqslant\|v\|_{L^{\infty}}$ for all $t>0$. Without loss of generality, assume $\|v\|_{L^{\infty}}=1$. Using the heat kernel, it is easily seen that there is a constant $C(\tau)$ such that

$$
\left\|D^{2} S(\tau / 2) v\right\|_{L^{\infty}} \leqslant C(\tau)<\infty .
$$

Now introduce the periodically extended version of the function $U(x):=\min \left(C(\tau)\|x\|^{2}, 1\right)$. Let $x_{*}$ be a point such that

$$
(S(\tau / 2) v))\left(x_{*}\right)=I_{*}=\inf _{\mathbb{R}^{n}}(S(\tau / 2) v)(\cdot) .
$$

Since $\langle S(\tau / 2) v\rangle=0$, we have $I_{*} \leqslant 0$. Consider $Q(x)=U\left(x-x_{*}\right)$. Then (22) and the definition of $U$ imply that $(S(\tau / 2) v)) \leqslant Q(x)$. Hence by the strong maximum principle, for all $t>0$ we have

$$
\sup _{x \in \mathbb{R}^{n}}(S(t+\tau / 2) v)(x) \leqslant \sup _{x \in \mathbb{R}^{n}}(S(t) Q)(x)=C_{1}(\tau)<1,
$$

which leads to 20 .

Proof of [21). This follows immediately from (20) once we observe that for any $t \in[k \tau,(k+1) \tau]$,

$$
\begin{aligned}
\|S(t) v\|_{L^{\infty}} & \leqslant\|S(k \tau) v\|_{L^{\infty}} \leqslant C_{1}(\tau)^{k}\|v\|_{L^{\infty}}=e^{-k\left|\ln \left(C_{1}(\tau)\right)\right|}\|v\|_{L^{\infty}} \\
& \leqslant e^{\left|\ln \left(C_{1}(\tau)\right)\right|} e^{-t\left|\ln \left(C_{1}(\tau)\right)\right| / \tau}\|v\|_{L^{\infty}}
\end{aligned}
$$

so that we can set $N=\left|\ln \left(C_{1}(\tau)\right)\right| / \tau$ and $M=e^{\left|\ln \left(C_{1}(\tau)\right)\right|}$.

What is left is proving the asymptotic statement $(9)$ in Theorem 2 about the propagating velocity. The strategy is to construct sub- and supersolutions of $(1)_{F}$ to provide sharp bounds for $T_{F}$. For this, we need to make use of the nondegeneracy assumption A3. In particular, this implies that there are only a finite number of stationary solutions $\left\{u^{(i)}\right\}_{i=1}^{N}$ of $\left\{14 F_{F_{*}}\right.$. Now, the key observation is that near each of the $u^{(i)}$ 's, the dynamical behaviour of the solution of $(1)_{F_{*}}$ is well-approximated by the dynamics on the centre-manifold while away from the stationary point, the velocity of the evolving graph is uniformly positive and independent of the additional forcing above the critical value. These are made precise by the following definitions and constructions.

\subsection{Construction of sub-and supersolutions near a stationary point}

Let $u_{*}$ be one of the solutions of $(14)_{F_{*}}$. By Proposition 5 5, 3), the principal eigenvalue $\lambda_{p}$ of the linearised operator (7) is zero and simple with positive eigenfunction $\varphi_{p}(x)$. The following construction is motivated by the centre-manifold theory for $(1)_{F_{*}+\gamma}$ near $u_{*}$ (see [15, pp. 173]). We make the following ansatz:

$$
v(x, t)=u_{*}(x)+\left(R_{0}(x)+s(t) \varphi_{p}(x)+R(x, t)\right)
$$

and compute:

$$
\begin{aligned}
v_{t}- & \Delta v-f(x, v)-F_{*}-\gamma=\dot{s}(t) \varphi_{p}+R_{t} \\
& \quad-\left\{L_{f}\left(u_{*}\right) R_{0}+\gamma+\frac{1}{2} f_{u u}\left(x, u_{*}\right)\left(R_{0}+s(t) \varphi_{p}+R\right)^{2}+L_{f}\left(u_{*}\right) R+E_{3}\left(R_{0}, s(t), R\right)\right\}
\end{aligned}
$$


where

$$
\left\|E_{3}\right\|_{\infty} \leqslant\left(|s|+\left\|R_{0}\right\|_{\infty}+\|R\|_{\infty}\right)^{2} \omega\left(|s|+\left\|R_{0}\right\|_{\infty}+\|R\|_{\infty}\right)
$$

for some continuous and increasing function $\omega(\cdot)$ such that $\omega(0)=0$. For simplicity, $\varphi_{p}$ is normalised to have norm one: $\left\|\varphi_{p}\right\|=1$. To construct the supersolution $v^{+}(x, t)$, we define $R_{0}, s$, and $R_{1}$ by

$$
\begin{aligned}
L_{f}\left(u_{*}\right) R_{0} & =-\gamma+\left\langle\gamma, \varphi_{p}\right\rangle \varphi_{p}, \\
\dot{s}(t) & =(1+\sigma)\left\{\left\langle\gamma, \varphi_{p}\right\rangle+\left\langle\frac{1}{2} f_{u u}\left(x, u_{*}\right)\left(s \varphi_{p}+R_{0}+R\right)^{2}, \varphi_{p}\right\rangle\right\}, \\
R_{t} & =L_{f}\left(u^{*}\right) R+\Psi, \quad R(0)=0, \\
\Psi & =\frac{1}{2} f_{u u}\left(x, u_{*}\right)\left(s \varphi_{p}+R_{0}+R\right)^{2}-\left\langle\frac{1}{2} f_{u u}\left(x, u^{*}\right)\left(s \varphi_{p}+R_{0}+R\right)^{2}, \varphi_{p}\right\rangle \varphi_{p},
\end{aligned}
$$

where $0<\sigma \ll 1$. The initial condition for $s$ is to be determined. With the above choices, $v^{+}$will be a supersolution if for all $x, t$,

$$
\left|E_{3}(x, t)\right| \leqslant \frac{1}{2} \sigma\left|\left\langle\gamma, \varphi_{p}\right\rangle \varphi_{p}(x)+\left\langle\frac{1}{2} f_{u u}\left(x, u_{*}\right)\left(R_{0}(x)+s(t) \varphi_{p}(x)+R(x, t)\right)^{2}, \varphi_{p}(x)\right\rangle\right| .
$$

Note that $\varphi_{p}$ is uniformly positive in the sense that $\varphi_{p}(x) \geqslant C_{p}>0$. To proceed further, we state the following two claims which are proved in Section 2.3

Claim I Assume A3. Then

$$
\int_{Q} f_{u u}\left(x, u_{*}(x)\right) \varphi_{p}(x)^{3} \mathrm{~d} x>0 .
$$

\section{CLAIM II Let}

$$
\mathcal{B}_{M, 0, T}:=\left\{(s, R):[0, T] \rightarrow \mathbb{R} \times L^{\infty}(Q): \sup _{t \in[0, T]}\left(|s(t)|+\|R(t)\|_{L^{\infty}}\right) \leqslant M\right\} .
$$

1. There exists $C_{0}>0$ such that

$$
\left\|R_{0}\right\|_{\infty} \leqslant C_{0} \gamma \text {. }
$$

2. There exist $0<\gamma_{0}, C_{1} \ll 1$ and $C, T_{*}>0$ such that for all $0<\gamma<\gamma_{0}$, there exists a solution $(s(t), R(t)) \in \mathcal{B}_{C_{1}, 0, T_{*}}$ of 26$\left.)-28\right)$ and

$$
\|R(t)\|_{\infty} \leqslant C\left(\sup _{r \in[0, t]} s(r)^{2}+\gamma^{2}\right) \quad \text { for } 0<t<T .
$$

Furthermore, $T_{*}$ can be chosen to be

$$
T_{*}=\inf \left\{t \geqslant 0:|s(t)| \geqslant C_{1}\right\}
$$

The above two claims imply that for $\gamma$ and $s$ small enough, we have

$$
\left|\left\langle\frac{1}{2} f_{u u}\left(x, u_{*}\right)\left[\left(R_{0}+s \varphi_{p}+R\right)^{2}-\left(s \varphi_{p}\right)^{2}\right], \varphi_{p}\right\rangle\right| \leqslant o(1)\left\{\left\langle\gamma, \varphi_{p}\right\rangle+\left\langle\frac{1}{2} f_{u u}\left(x, u_{*}\right) \varphi_{p}^{2}, \varphi_{p}\right\rangle s^{2}\right\} .
$$

This leads to the validity of 29 for the same range of $\gamma$ and $s$. Furthermore, we have

$$
\begin{aligned}
& (1+\sigma / 2)\left(\left\langle\gamma, \varphi_{p}\right\rangle+\left\langle\frac{1}{2} f_{u u}\left(x, u_{*}\right) \varphi_{p}^{2}, \varphi_{p}\right\rangle s(t)^{2}\right) \\
& \quad \leqslant \dot{s}(t) \leqslant(1+3 \sigma / 2)\left(\left\langle\gamma, \varphi_{p}\right\rangle+\left\langle\frac{1}{2} f_{u u}\left(x, u_{*}\right) \varphi_{p}^{2}, \varphi_{p}\right\rangle s^{2}(t)\right),
\end{aligned}
$$


which in particular implies that $\dot{s}(t)>0$ for $0<t<T_{*}$. Finally, by Claim II, there exists $0<K$ $<C_{1}$ independent of $\gamma$ such that for $0<\gamma<\gamma_{0}$ we have

$$
\frac{1}{2} K \varphi_{p}(x) \geqslant\left|R_{0}(x)+R(x, t)\right|
$$

for all $t$ as long as (31) holds.

DEFINITION 8 Given $0<\gamma, \sigma \ll 1$, the supersolution $v_{\text {in }}^{+}(x, t)$ near a stationary point $u_{*}$ is defined by (23), together with 25]-228):

$$
s(t): 0<t<T_{\text {in }}, \quad \text { where } s(0)=-K \text { and } s\left(T_{\text {in }}\right)=K .
$$

The subsolution $v_{\text {in }}^{-}(x, t)$ is defined in the same way except with the factor $1+\sigma$ in 26 replaced by $1-\sigma$. (By abuse of notation, we use the same $T_{\text {in }}$ for both the sub- and supersolutions, even though they can be two different values.)

Note that in both cases,

$$
v_{\text {in }}^{ \pm}(x, 0)<u_{*}(x)-\frac{1}{2} K C_{p} \quad \text { and } \quad v_{\text {in }}^{ \pm}\left(x, T_{\text {in }}\right)>u_{*}(x)+\frac{1}{2} K C_{p} .
$$

\subsection{Construction of sub-and supersolution away from a stationary point}

Now, by assumption, we have only finitely many (up to vertical translations) periodic solutions of 14 $F_{*}: u_{*}^{(1)}, \ldots, u_{*}^{(N)}$. By Proposition 5 2, they can be well-ordered: $u_{*}^{(1)}<\cdots<u_{*}^{(N)}$.

We now describe the construction of subsolutions between any two stationary solutions $u_{*}^{(i)}$ and $u_{*}^{(i+1)}$. (Supersolution can be trivially constructed upon the introduction of discontinuous jumps.) Let $v_{\mathrm{in}, i}^{-}$be the subsolutions near the $u_{*}^{(i)}$, s constructed in the previous section. Let also $\left[0, T_{\mathrm{in}, i}\right]$ be the time interval defined in (34) for $u_{*}^{(i)}$. Denote by $S_{0, t}^{*}(v)$ the solution at time $t$ of $(1) F_{*}$ with initial datum $v$ at time 0 . It has the following property which will be proved in Section 2.3 .

CLAIM III There exists a time $T>0$ bounded independently of $\gamma$ and $\sigma$ such that

$$
u_{*}^{(i+1)}-\frac{1}{2} K C_{p}<S_{0, T}^{*}\left(v_{\mathrm{in}, i}^{-}\left(T_{\mathrm{in}, i}\right)\right)<u_{*}^{(i+1)}
$$

where $K, C_{p}$ are the constants for the $u_{*}^{(i)}$ as in 35 .

We now give the following definition:

DEFINITION 9 The subsolution $v_{\text {out }, i, i+1}^{-}$between $u_{*}^{(i)}$ and $u_{*}^{(i+1)}$ is given by

where

$$
v_{\mathrm{out}, i, i+1}^{-}(x, t):=S_{0, t}^{*}\left(v_{\mathrm{in}, i}^{-}\left(\cdot, T_{\mathrm{in}, i}\right)\right) \quad \text { for } 0<t \leqslant T_{\mathrm{out}, i, i+1}
$$

$$
T_{\text {out }, i, i+1}:=\inf \left\{t: v_{\text {out }, i, i+1}^{-}(\cdot, t)-v_{\text {in }, i+1}^{-}(\cdot, 0) \geqslant 0\right\} .
$$

Note that the $v_{\text {out }, i, i+1}^{ \pm}$and $T_{\text {out }, i, i+1}$ do not depend on $\gamma$.

With the above construction, we can define the following sub- and supersolutions from $u_{*}^{(1)}(x)$ to $u_{*}^{(1)}(x)+1$, i.e. they evolve vertically through one spatial period. They are used to provide upper and lower bounds for the propagating speed of the front. 
DEFINITION 10 The subsolution $V^{-}(t)$ is given by

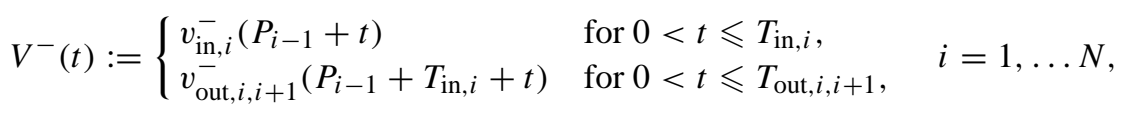

where $P_{0}=0$ and $P_{i}=\sum_{j=1}^{i}\left(T_{\mathrm{in}, j}+T_{\mathrm{out}, j, j+1}\right)$.

The supersolution $V^{+}(t)$ is given by

$$
V^{+}(t):=v_{\mathrm{in}, i}^{+}\left(Q_{i-1}+t\right) \quad \text { for } 0<t \leqslant T_{\mathrm{in}, i}, i=1, \ldots N,
$$

where $Q_{0}=0$ and $Q_{i}=\sum_{j=1}^{i} T_{\mathrm{in}, j}$.

REMARK. Note that we do not need the construction of the supersolution in between two stationary solutions $v_{\text {out }, i, i+1}^{+}$as we introduce the upward discontinuous jumps at the $Q_{i}$ 's. The above sub- and supersolutions are piecewise continuous in time, the subsolution is upper semicontinuous and the supersolution lower semicontinuous. In such a case a comparison theorem between solutions and, respectively, sub- and supersolutions holds. For such a comparison theorem see e.g. [25].

The following statement gives the sharp asymptotic value for the propagation speed.

THEOREM 11 Let $T_{\text {tot }}$ be the time it takes for the solution to travel one period in space. Then for each $\sigma>0$, there exists a $\gamma_{0}(\sigma)$ such that for $0<\gamma<\gamma_{0}(\sigma)$, we have

$$
(1-\sigma) A \gamma^{-1 / 2} \leqslant T_{\text {tot }} \leqslant(1+\sigma) A \gamma^{-1 / 2}
$$

where

$$
A=\pi \sum_{i=1}^{N}\left\{\left(\int_{Q} \varphi_{p}^{(i)}(x) \mathrm{d} x\right)\left\langle\frac{1}{2} f_{u u}\left(x, u_{*}^{(i)}\right)\left(\varphi_{p}^{(i)}\right)^{2}, \varphi_{p}^{(i)}\right\rangle\right\}^{-1 / 2} .
$$

Proof. This is a simple consequence of (32) and the uniform bound for the $T_{\text {out }, i, i+1}$ 's which is independent of $\gamma$. Consider the supersolution, $V^{+}(t)$. Let

$$
A_{1}:=\int_{Q} \varphi_{p}^{(i)}(x) \mathrm{d} x \quad \text { and } \quad A_{2}:=\left\langle\frac{1}{2} f_{u u}\left(x, u^{(i)}\right)\left(\varphi_{p}^{(i)}\right)^{2}, \varphi_{p}^{(i)}\right\rangle .
$$

Then upon integrating [32, we have

$$
\begin{aligned}
& \frac{1}{(1+3 \sigma / 2) \sqrt{A_{1} A_{2} \gamma}}\left\{\tan ^{-1}\left(\sqrt{\frac{A_{2}}{A_{1} \gamma}} s(t)\right)-\tan ^{-1}\left(\sqrt{\frac{A_{2}}{A_{1} \gamma}} s(0)\right)\right\} \\
& \leqslant t \leqslant \frac{1}{(1+\sigma / 2) \sqrt{A_{1} A_{2} \gamma}}\left\{\tan ^{-1}\left(\sqrt{\frac{A_{2}}{A_{1} \gamma}} s(t)\right)-\tan ^{-1}\left(\sqrt{\frac{A_{2}}{A_{1} \gamma}} s(0)\right)\right\} .
\end{aligned}
$$

Choosing $s(0)=-K, s\left(T_{\mathrm{in}, i}\right)=K$ as in Definition 8 , for $0<\gamma \ll 1$ we have

$$
\frac{\pi}{(1+3 \sigma / 2) \sqrt{A_{1} A_{2} \gamma}} \leqslant T_{\mathrm{in}, i} \leqslant \frac{\pi}{(1+\sigma / 2) \sqrt{A_{1} A_{2} \gamma}} .
$$

A similar estimate applies to $T_{\mathrm{in}, i}$ for the case of subsolution. 
Estimate (38) then follows immediately upon summing over the $i$ 's:

$$
\sum_{i=1}^{N} T_{\mathrm{in}, i} \leqslant T_{\text {tot }} \leqslant \sum_{i=1}^{N} T_{\mathrm{in}, i}+T_{\text {out }, i, i+1} .
$$

REMARK. Assumption A3 is needed for the precise time asymptotics. If we are only interested in the existence of a piecewise subsolution without further information, then we can simply use

$$
v^{-}(x, t):=u_{*}(x)+\frac{\gamma t}{2\left\|\varphi_{p}\right\|_{\infty}} \varphi_{p}(x)
$$

for $|t|<T_{1}(\gamma)$, and continue this solution away from the stationary points by the evolution at $F_{*}$ as in (36).

\subsection{Proof of Claims I, II, and III}

Proof of Claim I. We argue by contradiction. Consider for $0<s \ll 1$ the functions

$$
\begin{aligned}
& w:=u_{*}+s \varphi_{p}-s^{2} R \\
& R=\left(L_{f}\left(u_{*}\right)\right)^{-1}\left(\frac{1}{2} f_{u u}\left(x, u_{*}\right) \varphi_{p}^{2}-\left\langle\frac{1}{2} f_{u u}\left(x, u_{*}\right) \varphi_{p}^{2}, \varphi_{p}\right\rangle \varphi_{p}\right) .
\end{aligned}
$$

Clearly $u_{*}$ is a supersolution of (14) $F_{*}+\gamma$ for any $\gamma>0$, and $u_{*}<w$ for $s$ small enough. If we can show that $w$ is a subsolution, then there exists a stationary solution for $F_{*}+\gamma$, which contradicts the assumption that $F_{*}$ is critical.

By expanding the nonlinearity up to second order we obtain

$$
\Delta w+f(x, w)+F_{*}+\gamma=s^{2}\left\langle\frac{1}{2} f_{u u}\left(x, u_{*}\right) \varphi_{p}^{2}, \varphi_{p}\right\rangle+o\left(s^{2}\right)+\gamma,
$$

which can be made negative by first choosing $s$ so small that the $o\left(s^{2}\right)$-terms are dominated by the second order terms, and then choosing $\gamma$ to be sufficiently small.

Proof of Claim II. 1. The right hand side of 25] is orthogonal to $\varphi_{p}$. Hence there exists $c>0$ such that $\left\|R_{0}\right\|_{2} \leqslant c \gamma$. The regularity assumption $\mathbf{A 1}$ for the nonlinearity implies further that $\left\|R_{0}\right\|_{W^{2,2}} \leqslant$ $c^{\prime \prime} \gamma$. The claim follows directly (for $n<4$ ) or from $\left\|R_{0}\right\|_{p} \leqslant c^{\prime \prime \prime} \gamma$ for some $p>0$. We can then conclude by a standard bootstrapping procedure.

2. The local existence for $R(\cdot, t) \in L^{2}(0,1)$ follows from a Banach fixed point type argument. To prove (31), let $\Psi$ be as in 28). Then by Step 1, we can find a $T$ and $(s, R) \in \mathcal{B}_{C_{1}, 0, T}$ for any $C_{1}$, provided the initial datum satisfies $\|R(0)\|+|s| \leqslant C_{1} / 2$. Given a small constant $\rho$, we can choose $C_{1}$ sufficiently small in order to ensure that there exists a large constant $C_{3}(\rho)$ such that

$$
|\Psi(x, t)| \leqslant \rho R(x, t)+C\left(\gamma^{2}+s(t)^{2}\right)
$$

Moreover, there exist $A>0$ and $b>0$ such that if $\left\langle R, \varphi_{p}\right\rangle=0$, then

$$
\left\|e^{t L_{f}\left(u_{*}\right)} R\right\|_{\infty} \leqslant A e^{-b t}\|R\|_{\infty} .
$$

The above is proved by first establishing that $\left\|e^{L_{f}\left(u_{*}\right)} R\right\|_{\infty} \leqslant M\|R\|_{L^{2}}$ for some $M>0$. As standard regularity theory for the periodic Laplacian implies that $u_{*}$ is at least $C^{2}$, this follows 
from short time regularity estimates for parabolic equations (see for example [12, Chapter 1.6, Thm. 11], adapted to the periodic case, for Hölder continuous coefficients, or for an alternative approach [15, Chapter 3.5, Exercise 4] which requires less regularity assumption for the potential). Then (41) follows from the $L^{2}$-exponential decay in time due to the spectral gap for $L_{f}\left(u_{*}\right)$.

Hence we can estimate

$$
\begin{aligned}
\|R(t)\|_{\infty} & \leqslant \int_{0}^{t} A e^{-b\left(t-t^{\prime}\right)}\left(\rho\left\|R\left(t^{\prime}\right)\right\|_{\infty}+C\left(\gamma^{2}+s\left(t^{\prime}\right)^{2}\right) \mathrm{d} t^{\prime}\right. \\
& \leqslant \frac{A}{b}\left(\rho \sup _{t^{\prime} \in[0, t]}\left\|R\left(\cdot, t^{\prime}\right)\right\|_{\infty}+\sup _{t^{\prime} \in[0, t]}\left|s\left(t^{\prime}\right)\right|+\gamma^{2}\right) .
\end{aligned}
$$

The claim follows if $\rho$ is chosen so small that $A b^{-1} \rho \leqslant 1 / 2$.

Proof of Claim III. Let $v(x):=v_{\mathrm{in}, i}^{-}\left(x, T_{\mathrm{in}}\right)$. Consider the sequence of functions

$$
v_{n}:=\left(S_{0, n}^{*}\right)(v)
$$

As $u_{*}^{(i)}<v_{n} \leqslant u_{*}^{(i+1)}$, the sequence is bounded in $L^{\infty}$; then parabolic regularity (recall the regularity assumption A1) implies that it is bounded in $C^{0, \alpha}$ for some $\alpha>0$. Hence it has a convergent subsequence in $C^{0}$. This sequence converges to either $u_{*}^{(i)}$ or $u_{*}^{(i+1)}$.

It remains to show that $v_{n}$ cannot converge to $u_{*}^{(i)}$. Let $w$ be as in (40). By expanding the nonlinearity as in the proof of Claim I, we conclude that $w$ is a supersolution of $14 F_{*}$ for $s$ sufficiently small. Moreover we can choose $w$ in such a way that $u_{*}^{(i)}<w<v$. Therefore, convergence of $v_{n}$ to $u_{*}^{(i)}$ contradicts the comparison principle.

By the same reasoning, $\left(S_{0, t}^{*}\right)(w)$ will converge to $u_{*}^{(i+1)}$. This behaviour does not depend on $\gamma$ and $\sigma$ and hence the time it takes for $w$ to evolve from $u_{*}^{(i)}+\frac{1}{2} K C_{p}$ to $u_{*}^{(i+1)}-\frac{1}{2} K C_{p}$ is independent of them. The claim follows as $S_{0, t}^{*}(w) \leqslant S_{0, t}^{*}(v)$.

\section{$2.4 \quad$ Nondegeneracy condition $\mathbf{A 3}$}

This section demonstrates that there are "abundant" examples of nonlinear functions $f(x, u)$ satisfying the nondegeneracy condition A3. See also Section 4.1 for an explicit example.

Proposition 12 Given any $f(x, u)$ satisfying $\mathbf{A} \mathbf{1}$ and $\mathbf{A} \mathbf{2}$ and any $\epsilon>0$, there exists a function $g(x, u)$ satisfying A1-A3 with $\|g-f\|_{\infty}<\epsilon$. Moreover, $g$ can be chosen such that:

1. The critical forces for $f$ and $g$ are the same.

2. The set $\left\{u: \Delta u+g(x, u)+F_{*}=0\right\}$ can be taken to be any finite subset of $\left\{u: \Delta u+f(x, u)+F_{*}=0\right\}$.

3. Instead of property 1 above, we can require that

$$
\int_{0}^{1} \int_{[0,1]^{n}} g(x, u) \mathrm{d} x \mathrm{~d} u=\int_{0}^{1} \int_{[0,1]^{n}} f(x, u) \mathrm{d} x \mathrm{~d} u
$$

(so that Lemma 4 can be applied to $g$ ). In this case, the critical forces for $g$ and $f$ can differ by at most order $\epsilon$. 
Proof. Given any finite subset of $\left\{u: \Delta u+f(x, u)+F_{*}=0\right\}=\left\{u^{(1)}<\cdots<u^{(N)}\right\}$ (and setting $u^{(j+N)}(x)=u^{(j)}(x)+1$ for $\left.j \in \mathbb{Z}\right)$ we introduce

$$
g(x, u)=f(x, u)+\epsilon \prod_{j=-\infty}^{\infty} Q\left(\frac{u-u^{(j)}(x)}{\frac{1}{8} \min _{j}\left\|u^{(j+1)}-u^{(j)}\right\|_{\infty}}\right)
$$

where $Q$ is a smooth even function having the form

$$
Q(r)=\left\{\begin{array}{ll}
\frac{1}{2} r^{2} & \text { for }|r| \leqslant 1, \\
1 & \text { for }|r| \geqslant 2,
\end{array} \text { and } Q(|r|)\right. \text { is an increasing function, }
$$

so that $Q(0)=Q^{\prime}(0)=0$ and $Q^{\prime \prime}(0)>0$.

Let $F_{*}$ be the critical force for $f$. As $g \geqslant f$, the critical force for $g$ is no greater than $F_{*}$. On the other hand, it is immediate that

$$
\left\{u^{(j)}\right\}_{j=-\infty}^{\infty} \subset\left\{u: \Delta u+g(x, u)+F_{*}=0\right\} .
$$

Thus $g$ and $f$ have the same critical force value.

Analogous to (7), consider the linearised operator

$$
L_{g}\left(u^{(i)}\right) \varphi=\Delta \varphi+\partial_{u} g\left(x, u^{(i)}\right) \varphi .
$$

Let $\varphi_{p}^{(i)}(x)(>0)$ be the principal eigenfunction of $L_{f}\left(u^{(i)}\right)$. Note that

$$
L_{g}\left(u^{(i)}\right) \varphi_{p}^{(i)}=L_{f}\left(u^{(i)}\right) \varphi_{p}^{(i)}+\left.\epsilon\left\{\prod_{j=-\infty}^{\infty} Q\left(\frac{u-u^{(j)}(x)}{\frac{1}{8} \min _{j}\left\|u^{(j+1)}-u^{(j)}\right\|_{\infty}}\right)\right\}^{\prime}\right|_{u=u^{(i)}} \varphi_{p}^{(i)}=0 .
$$

Hence it is also the principal eigenfunction of $L_{g}\left(u^{(i)}\right)$ with eigenvalue 0 . Moreover,

$$
\int_{Q} \partial_{u}^{2} g\left(x, u^{(i)}(x)\right) \varphi_{p}(x)^{3} \mathrm{~d} x=\int_{Q} \partial_{u}^{2} f\left(x, u^{(i)}(x)\right) \varphi_{p}(x)^{3} \mathrm{~d} x+\epsilon \int_{Q} \varphi_{p}(x)^{3} \mathrm{~d} x>0 .
$$

Thus $\mathbf{A 3}$ is satisfied for $g$ at any $u^{(i)}$.

To continue, suppose $\Delta v+g(x, v)+F_{*}=0$ and $v$ is not equal to any of the $u^{(i)}$ 's. Then we must have $\Delta v+f(x, v)+F_{*} \leqslant 0$ but not identically zero. Now, similar to the proof of Proposition 5]2), consider

$$
u_{t}=\Delta u+f(x, u)+F_{*}, \quad u(x, 0)=v(x) .
$$

There exist $\gamma, t_{*}>0$ such that $\Delta u\left(x, t_{*}\right)+f\left(x, u\left(x, t_{*}\right)\right)+F_{*}+\gamma<0$, i.e. $u\left(x, t_{*}\right)$ is a subsolution of $14 F_{*}+\gamma$. Combining this with the fact that each $u^{(i)}$ is a supersolution of $14 F_{*}+\gamma$, we get a contradiction with the definition of $F_{*}$. Thus $\left\{u: \Delta u+g(x, u)+F_{*}=0\right\} \subset\left\{u^{(J)}\right\}_{j=-\infty}^{\infty}$.

Finally, by assumption, for $f(x, u)$, there is a stable solution of $(14)_{0}$ in the sense of $\mathbf{A 2}$. By the implicit function theorem, for any small enough $\epsilon>0$, the same condition also holds for $g(x, u)$.

Statement 3 follows simply by changing $g$ to $g(x, u)-\int_{0}^{1} \int_{[0,1]^{n}} g(x, u) \mathrm{d} x \mathrm{~d} u$.

Proposition 13 Given any collection $\left\{u^{(1)}<\cdots<u^{(N)}\right\}$ of $\mathbb{Z}^{n}$-periodic functions (and setting $u^{(j+N)}(x)=u^{(j)}(x)+1$ for $\left.j \in \mathbb{Z}\right)$, there exists an $f(x, u)$ satisfying $\int_{0}^{1} \int_{[0,1]^{n}} f(x, u) \mathrm{d} x \mathrm{~d} u=0$ and conditions A1-A3 such that at its critical forcing $F_{*}$, we have

$$
\left\{u: \Delta u+f(x, u)+F_{*}=0\right\}=\left\{u^{(1)}<\cdots<u^{(N)}\right\} .
$$


Proof. The proof is similar to that of the previous proposition once we take $f$ to be $\tilde{f}(x, u)-$ $\int_{0}^{1} \int_{[0,1]^{2}} \tilde{f} \mathrm{~d} x \mathrm{~d} u$ where

$$
\begin{aligned}
\tilde{f}(x, u)= & -\prod_{j=-\infty}^{\infty} \Delta u^{(j)}(x) P\left(\frac{u-u^{(j)}(x)}{\frac{1}{8} \min _{j}\left\|u^{(j+1)}-u^{(j)}\right\|_{\infty}}\right) \\
& +A \prod_{j=-\infty}^{\infty} Q\left(\frac{u-u^{(j)}(x)}{\frac{1}{8} \min _{j}\left\|u^{(j+1)}-u^{(j)}\right\|_{\infty}}\right)
\end{aligned}
$$

and

- $A \gg\left\{\max _{i}\left\|\Delta u^{(i)}\right\|_{\infty}\right\}$

- $P$ is a smooth positive even function such that $P(r)=1$ for $|r|<1$ and $P(r)=0$ for $|r|>2$;

- $Q$ is as in 42,

With the above $f$, for large enough $A$, in fact, we have $F_{*}=\iint \tilde{f} \mathrm{~d} x \mathrm{~d} u$. First of all, it is simple to check that

$$
\left\{u^{(j)}\right\}_{j=-\infty}^{\infty} \subset\left\{u: \Delta u+f(x, u)+F_{*}=0\right\} .
$$

In addition, at $F_{*}$ and for each $u^{(i)}$, the linearised operator $L_{f}\left(u^{(i)}\right)$ has the principal eigenvalue $\lambda_{p}=0$ and eigenfunction $\varphi_{p} \equiv 1$. Furthermore, as $\partial_{u}^{2} f\left(x, u^{(i)}\right) \equiv 1 / 2>0, \mathbf{A} 3$ is satisfied. Also, the facts that $\left\{u: \Delta u+f(x, u)+F_{*}=0\right\} \subset\left\{u^{(j)}\right\}_{j=-\infty}^{\infty}$ and $F_{*}$ is indeed the critical force follow from the following one-parameter family of supersolutions of $\left(14 F_{*}\right.$ "foliating" the region between any $u^{(j)}$ and $u^{(j+1)}$ :

$$
v_{\lambda}(x)= \begin{cases}u^{(j)}(x)+d \lambda & \text { for } 0<\lambda \leqslant 1 \\ (2-\lambda)\left(u^{(j)}(x)+d\right)+(\lambda-1)\left(u^{(j+1)}(x)-d\right) & \text { for } 1<\lambda \leqslant 2 \\ u^{(j+1)}(x)-d(3-\lambda) & \text { for } 2<\lambda \leqslant 3\end{cases}
$$

where $d=\frac{1}{8} \min _{j}\left\|u^{(j+1)}-u^{(j)}\right\|_{\infty}$. (Recall that we have chosen $A$ to be sufficiently large.)

Since $\iint f \mathrm{~d} x \mathrm{~d} u=0$, we can apply Proposition 4 and the remark afterward to ensure that $\mathbf{A 2}$ is also satisfied.

\section{Forced Allen-Cahn equation in the perturbative regime}

The equation studied in this section is the spatially inhomogeneous Allen-Cahn equation (2) in the regime $0<\delta \ll 1$. We assume that $g(\cdot)$ is a $C^{3}$ spatial 1-periodic function: $g(x+1)=g(x)$.

In order to study the front propagation in heterogeneous medium, we only consider solutions resembling a front structure. For $0<\delta \ll 1$, the equation 2 has two end states close to 1 and -1 . These are described by means of the functions $m^{ \pm}(x)$ which satisfy

$$
\begin{aligned}
\delta m_{x x}^{-}(x)-W^{\prime}\left(1+\delta m^{-}(x)\right)+\delta(g(x)+F) & =0, \\
\delta m_{x x}^{+}(x)-W^{\prime}\left(-1+\delta m^{+}(x)\right)+\delta(g(x)+F) & =0 .
\end{aligned}
$$

We now take the following ansatz for the solution $v(x, t)$ of (2):

$$
v(x, t)=m(z)+\delta E(z, t)+\delta \varphi(z, t)
$$


where $z=x-\delta c(t), E(z, t)=\alpha(z) m^{-}(x)+\beta(z) m^{+}(x)$ and $\alpha(z)$ and $\beta(z)$ are two smooth positive functions such that for some large but fixed positive constant $K$,

$$
\alpha(z)=\left\{\begin{array}{ll}
1 & \text { for } z<-K, \\
0 & \text { for } z>-K+1,
\end{array} \quad \beta(z)= \begin{cases}0 & \text { for } z<K-1, \\
1 & \text { for } z>K .\end{cases}\right.
$$

Note that the above ansatz for $v(x, t)$ connects $-1+\delta m^{+}(x)$ as $x \rightarrow+\infty$ and $1+\delta m^{-}(x)$ as $x \rightarrow-\infty$. Now $\varphi(z, t)$ satisfies an equation of the form

$$
\begin{aligned}
\varphi_{t}= & \varphi_{z z}+\dot{c}\left(m_{z}+\delta E_{z}+\delta \varphi_{z}\right)-E_{t} \\
& +\frac{1}{\delta}\left(m_{z z}+\delta E_{z z}-W^{\prime}(m+\delta E+\delta \varphi)+\delta(g(z+\delta c(t))+F)\right)
\end{aligned}
$$

so that

$$
\varphi_{t}=\varphi_{z z}-W^{\prime \prime}(m) \varphi+H(t, z, \varphi)
$$

where

$$
\begin{aligned}
H(t, z, \varphi)= & \dot{c}\left(m_{z}+\delta E_{z}+\delta \varphi_{z}\right)-E_{t} \\
& +\frac{1}{\delta}\left\{m_{z z}+\delta E_{z z}-W^{\prime}(m+\delta E)+\delta(g(z+\delta c(t))+F)\right\} \\
& +O_{1}(\delta \varphi)+O_{2}\left(\delta \varphi^{2}\right) .
\end{aligned}
$$

We will work with the following weighted function spaces:

$$
\begin{aligned}
& \mathcal{B}_{0, b}=\left\{f:\|f\|_{0, b}=\sup \left\{\left|f(z) e^{b|z|}\right|: z \in \mathbb{R}\right\}<\infty\right\}, \\
& \mathcal{B}_{1, b}=\left\{f:\|f\|_{1, b}=\|f\|_{0, b}+\left\|f^{\prime}\right\|_{0, b}\right\}
\end{aligned}
$$

where $0<b<W^{\prime \prime}( \pm 1)$. The value of $b$ will be chosen later according to the spectral estimates 83 .

Expressions (45) and (46) are estimated as follows. For (45),

$$
455=\dot{c}\left(m_{z}+\delta E_{z}+\delta \varphi_{z}\right)-E_{t}=\dot{c}\left\{m_{z}+\delta\left(\alpha^{\prime}(z) m^{-}(x)+\beta^{\prime}(z) m^{+}(x)\right)+\delta \varphi_{z}\right\}
$$

so that for some constant $A$,

$$
\|(45)\|_{0, b}<A|\dot{c}(t)|\left(1+\delta\|\varphi(\cdot, t)\|_{1, b}\right) .
$$

For 46 , it can be rewritten as

$$
\begin{aligned}
\text { 466) }=\frac{1}{\delta}\left\{m_{z z}-W^{\prime}(m+\delta E)+W^{\prime}(\alpha(z)\right. & +\delta E-\beta(z)) \\
& \left.+\delta E_{z z}-W^{\prime}(\alpha(z)+\delta E-\beta(z))+\delta(g(x)+F)\right\} .
\end{aligned}
$$

After some routine computation, it is easily verified that for some constant $A$ again,

$$
\|46\|_{0, b}<A .
$$

Hence

$$
\|H(\cdot, t)\|_{0, b} \leqslant A\left\{1+|\dot{c}(t)|\left(1+\delta\|\varphi(\cdot, t)\|_{1, b}\right)+\delta\|\varphi\|_{1, b}+\delta\|\varphi\|_{1, b}^{2}\right\} .
$$


We now choose $c(t)$ to make $\int \varphi(z, t) m_{z}(z) \mathrm{d} z=0$ for all $t>0$. Such a choice can always be made if $\int \varphi(z, 0) m_{z}(z) \mathrm{d} z=0$ and $c(t)$ satisfies

$$
\dot{c}(t)=-\frac{1}{\delta} \frac{\left\langle m_{z z}+\delta E_{z z}-W^{\prime}(m+\delta E)+\delta(g(z+\delta c(t))+F), m_{z}\right\rangle}{\left\|m_{z}\right\|^{2}+\delta\left\langle\alpha^{\prime}(z) m^{-}(x)+\beta^{\prime}(z) m^{+}(x)+\varphi_{z}, m_{z}\right\rangle} .
$$

The above expression is well defined provided that the denominator of the right hand side does not vanish. In particular, there exist some positive constants $B, C$ such that

$$
\text { if } \quad C>\delta\|\varphi\|_{1, b}, \quad \text { then } \quad|\dot{c}(t)| \leqslant \frac{B}{C-\delta\|\varphi(\cdot, t)\|_{1, b}} .
$$

Such a choice of $c(t)$ also implies that

$$
\int H(z, t) m_{z}(z) \mathrm{d} z=0 \quad \text { for all } t \geqslant 0 .
$$

Next we will make use of the decay properties of the linear operator

$$
L_{W} \psi=\psi_{z z}-W^{\prime \prime}(m(z)) \psi
$$

to deduce some long time estimates for $\|\varphi(\cdot, t)\|_{1, b}$. First we provide the following preliminary result.

LEMMA 14 (Gronwall type estimate) Let $f(t)(t \geqslant 0)$ be a continuous positive function such that for some constants $P \geqslant 1, Q, R, \omega$, and $\delta>0$,

$$
f(t) \leqslant P e^{-\omega t} f(0)+\int_{0}^{t} e^{-\omega(t-s)}(Q+R \delta f(s)) \mathrm{d} s .
$$

If further $R \delta \leqslant \omega / 2$, then $f(t) \leqslant P f(0)+2 Q / \omega$ for all $t \geqslant 0$.

Proof. Let $h(t)=e^{\omega t} f(t)$. Then

$$
h(t) \leqslant P h(0)+\frac{Q}{\omega}\left(e^{\omega t}-1\right)+R \delta \int_{0}^{t} h(s) \mathrm{d} s
$$

Gronwall's inequality implies that

$$
\begin{aligned}
h(t) & \leqslant P h(0)+\frac{Q}{\omega}\left(e^{\omega t}-1\right)+R \delta \int_{0}^{t} e^{R \delta(t-s)}\left(P h(0)+\frac{Q}{\omega}\left(e^{\omega s}-1\right)\right) \mathrm{d} s \\
& \leqslant P h(0)+\frac{Q}{\omega}\left(e^{\omega t}-1\right)+P h(0)\left(e^{R \delta t}-1\right)+\frac{Q R \delta}{\omega(\omega-R \delta)}\left(e^{\omega t}-e^{R \delta t}\right)+\frac{Q}{\omega}\left(1-e^{R \delta t}\right) .
\end{aligned}
$$

Hence switching back to $f(t)$ and upon simplification, we get

$$
f(t) \leqslant P f(0) e^{-(\omega-R \delta) t}+\frac{Q}{\omega-R \delta}\left(1-e^{-(\omega-R \delta) t}\right),
$$

leading to the desired statement. 
Now the solution $\varphi(\cdot, t)$ of (44) can be written as

$$
\varphi(t)=S(t) \varphi(0)+\int_{0}^{t} S(t-s) H(s) \mathrm{d} s
$$

where $S(t)$ is the solution operator for the linear equation $\varphi_{t}=L_{W} \varphi$. We have the following estimate for $\varphi(t)$ :

Proposition 15 There exist $D>1$ and $\omega>0$ such that if $\sup _{s \in[0, t]} \delta\|\varphi(s)\|_{1, b}<C / 2$, then

$$
\begin{aligned}
\|\varphi(t)\|_{1, b} \leqslant D e^{-\omega t}\|\varphi(0)\|_{1, b}+\int_{0}^{t} D e^{-\omega(t-s)} A\left\{1+\frac{2 B}{C}\left(1+\delta\|\varphi(s)\|_{1, b}\right)\right. & \\
& \left.+\delta\|\varphi(s)\|_{1, b}+\delta\|\varphi(s)\|_{1, b}^{2}\right\} \mathrm{d} s .
\end{aligned}
$$

Proof. From [55, we can write

$$
\|\varphi(t)\|_{1, b} \leqslant\left\|S(t) \varphi_{0}\right\|_{1, b}+\int_{0}^{t}\|S(t-s) H(s)\|_{1, b} \mathrm{~d} s .
$$

The estimate for $S(t)$ (from Theorem 25 gives $\|S(t) \varphi(0)\|_{1, b} \leqslant D e^{-\omega t}\left\|\varphi_{0}\right\|_{1, b}$. To estimate the remaining term, consider, for $0<t<2$,

$$
\int_{0}^{t}\|S(t-s) H(s)\|_{1, b} \mathrm{~d} s \leqslant \int_{0}^{t} \frac{D e^{-\omega(t-s)}}{\sqrt{t-s}}\|H(s)\|_{0, b} \mathrm{~d} s \leqslant C\|\varphi(0)\|_{1, b}
$$

where we have made use of 80 . For $t>2$, consider

$$
\begin{aligned}
\int_{0}^{t}\|S(t-s) H(s)\|_{1, b} \mathrm{~d} s & \leqslant \int_{0}^{t-1}\|S(t-s) H(s)\|_{1, b} \mathrm{~d} s+\int_{t-1}^{t}\|S(t-s) H(s)\|_{1, b} \mathrm{~d} s \\
& \leqslant \int_{0}^{t-1} \frac{D e^{-\omega(t-s)}}{\sqrt{t-s}}\|H(s)\|_{0, b} \mathrm{~d} s+\int_{t-1}^{t} D e^{-\omega(t-s)}\|H(s)\|_{1, b} \mathrm{~d} s .
\end{aligned}
$$

By (50) and (52), the first integral can be bounded by the term stated in the proposition. For the second integral, an extension of (50) and parabolic regularity as in (67) give

$$
\begin{array}{rl}
\int_{t-1}^{t} & D e^{-\omega(t-s)}\|H(s)\|_{1, b} \mathrm{~d} s \\
& \leqslant \int_{t-1}^{t} D e^{-\omega(t-s)} A\left\{1+|\dot{c}(s)|\left(1+\delta\|\varphi(s)\|_{2, b}\right)+\delta\|\varphi(s)\|_{2, b}+\delta\|\varphi(s)\|_{2, b}^{2}\right\} \mathrm{d} s \\
& \leqslant \int_{t-1}^{t} D^{\prime} e^{-\omega(t-s)} A\left\{1+\frac{2 B}{C}\left(1+\delta\|\varphi(s-1)\|_{1, b}\right)+\delta\|\varphi(s-1)\|_{1, b}+\delta\|\varphi(s-1)\|_{1, b}^{2}\right\} \mathrm{d} s \\
& \leqslant \int_{t-2}^{t-1} D^{\prime} e^{-\omega(t-s)} A\left\{1+\frac{2 B}{C}\left(1+\delta\|\varphi(s)\|_{1, b}\right)+\delta\|\varphi(s)\|_{1, b}+\delta\|(s)\|_{1, b}^{2}\right\} \mathrm{d} s,
\end{array}
$$

which can then be absorbed into the first integral. The stated conclusion is thus proved. 
From the above result, we see that if for some $T>0, \varphi$ satisfies $\sup _{t \in[0, T]}\|\varphi(s)\|_{1, b} \leqslant M$ (with $M$ to be chosen later) then for $\delta \leqslant C / 2 M$, there are positive constants $E$ (independent of $M$ ) and $F_{M}$ such that

$$
\|\varphi(t)\|_{1, b} \leqslant D e^{-\omega t}\|\varphi(0)\|_{1, b}+\int_{0}^{t} e^{-\omega(t-s)}\left(E+F_{M} \delta\|\varphi(s)\|_{1, b}\right) \mathrm{d} s \quad \text { for } 0 \leqslant t \leqslant T .
$$

The next two results provide some long time estimates for the solution $\varphi(t)$. In all of the following, the constants $A, B, C, D, E, F_{M}, \omega$ are as in 507, 52) and (56).

Proposition 16 Let $M \geqslant 8 E / \omega$ and $\delta<\min \left\{\omega / 2 F_{M}, C / 2 M\right\}$. If the initial condition satisfies $\|\varphi(0)\|_{1, b} \leqslant M / 4 D$, then $\|\varphi(t)\|_{1, b} \leqslant M / 2$ for all $t>0$.

Proof. Suppose $T_{M}=\inf \left\{t:\|\varphi(t)\|_{1, b}=M\right\}<\infty$. Then Lemma 14 says

$$
\sup _{t \in\left[0, T_{M}\right]}\|\varphi(t)\|_{1, b} \leqslant D\|\varphi(0)\|_{1, b}+\frac{2 E}{\omega} \leqslant \frac{M}{4}+\frac{M}{4} \leqslant \frac{M}{2} .
$$

Then standard parabolic regularity implies that $\|\varphi(t)\|_{1, b} \leqslant M$ for $t \in\left[T_{M}, T_{M}+\epsilon\right]$ where $\epsilon$ is some small enough number. This contradicts the definition of $T_{M}$. Hence $T_{M}=\infty$ and so $\|\varphi(t)\|_{1, b} \leqslant$ $M / 2$ for all $t \geqslant 0$.

Proposition 17 Let $M \geqslant 16 D E / \omega$ and $\delta \leqslant \min \left\{\omega / 8 D F_{M}, C / 2 M\right\}$. If $\|\varphi(0)\|_{1, b} \leqslant M / 4 D$, then $\|\varphi(t)\|_{1, b} \leqslant M / 4 D$ for all $t \geqslant \omega^{-1} \ln (2 D)$.

Proof. Note that under the current hypothesis, the assumptions of the previous Proposition 16 are also satisfied. Hence we can legitimately write

$$
\begin{aligned}
\|\varphi(t)\|_{1 . b} & \leqslant D e^{-\omega t}\|\varphi(0)\|_{1, b}+\int_{0}^{t} e^{-\omega(t-s)}\left(E+\frac{F_{M} \delta M}{2}\right) \mathrm{d} s \leqslant \frac{M}{4} e^{-\omega t}+\frac{1}{\omega}\left(E+\frac{1}{2} F_{M} \delta M\right) \\
& \leqslant \frac{M}{8 D}+\frac{E}{\omega}+\frac{F_{M} \delta}{2 \omega} M \leqslant \frac{M}{8 D}+\frac{M}{16 D}+\frac{M}{16 D}=\frac{M}{4 D} .
\end{aligned}
$$

The next quantitative result implies that as long as the external forcing $F$ is near the threshold value, it takes a very long time for the front to travel across the heterogeneous spatial environment. Thus, the condition of large time in Proposition 17 is automatically satisfied. Recall the definitions given in $(10)$.

Theorem 18 (Estimation of velocity) Let $M \geqslant 16 D E / \omega$. Then for any $\sigma>0$, there exist $0<$ $\delta_{*} \leqslant \min \left\{\omega / 8 D F_{M}, C / 2 M\right\}$ and $C_{1}, C_{2}>0$ such that for any $0<\delta<\delta_{*}$ and $\left\|\varphi_{0}\right\|_{1, b} \leqslant M / 4 D$ and the forcing $F$ within the range

$$
0<C_{1} \delta \leqslant F-F_{*} \leqslant C_{2},
$$

the velocity $V_{F}$ of the front satisfies the estimate (13).

Proof. From [51, as $\|\varphi(t)\|_{1, b} \leqslant M / 2$ for all $t$, we see that

$$
\begin{aligned}
\dot{c}(t) & =\frac{1}{\delta} \frac{\left\langle m_{z z}+\delta E_{z z}-W^{\prime}(m)-\delta W^{\prime \prime}(m) E+O\left(\delta^{2}\right)+\delta(g(z+\delta c(t))+F),-m_{z}\right\rangle}{\left\|m_{z}\right\|^{2}+O(\delta)} \\
& =\frac{\left\langle g(z+\delta c(t))+F,-m_{z}\right\rangle}{\left\|m_{z}\right\|^{2}}+O(\delta)
\end{aligned}
$$

where we have used the facts that $m_{z z}-W^{\prime}(m)=0$ and $m_{z z z}-W^{\prime \prime}(m) m_{z}=0$. 
Using the notations of (10), we can write

$$
\begin{aligned}
\dot{c}(t) & =\frac{\left.\left(2 F+h_{*}\right)+(h(\delta c(t)))-h_{*}\right)}{\left\|m_{z}\right\|^{2}}+O(\delta) \\
& =\alpha\left(\gamma+O(\delta)+\beta(\delta c(t))^{2}\right), \quad \text { where } \gamma=F+h_{*} / 2 .
\end{aligned}
$$

Now fix a positive constant $0<P \ll 1$ which is independent of $\gamma$ and $\delta$. Then the time $t_{P}$ it takes for the front location $\delta c(t)$ to travel from 0 to $P$ can be computed as

$$
t_{P}=\frac{1}{\alpha} \int_{0}^{P / \delta} \frac{\mathrm{d} c}{\gamma+O(\delta)+\beta(\delta c)^{2}} .
$$

Similar to the proof of Theorem 11 , by choosing $C_{1}$ large enough and $\delta_{*}$ and $C_{2}$ small enough, we can estimate $t_{P}$ as

$$
\frac{(1-\sigma) \pi}{2 \alpha \delta \sqrt{\beta \gamma}} \leqslant t_{P} \leqslant \frac{(1+\sigma) \pi}{2 \alpha \delta \sqrt{\beta \gamma}} .
$$

Again, the time it takes for the front to travel from $P$ to $1-P$ is some number independent of $\gamma$ and $\delta$. We conclude that the total time $T_{\gamma}$ for the front to travel one period of the spatial heterogeneity follows the same asymptotics as $t_{P}$, leading to the assertion of the theorem.

The previous results facilitate the use of the Schauder fixed point theorem to prove the existence of a pulsating wave.

THEOREM 19 (Existence of fixed point) For any $M, \delta$ and $F$ as in Theorem 18 , there exists a $\varphi_{0}$ with $\left\|\varphi_{0}\right\|_{1, b} \leqslant M / 4 D$ and $0<T\left(\varphi_{0}\right)<\infty$ such that

$$
\varphi\left(T\left(\varphi_{0}\right)\right)=\varphi_{0}
$$

where $\varphi(t)$ is the solution of (44) with initial data $\varphi_{0}$.

Proof. Let $(\varphi(t), c(t))$ be the solution of (44) and $(51)$ with initial condition $\left(\varphi_{0}, c_{0}\right)$. In addition, let

$$
T\left(\varphi_{0}\right)=\inf \left\{t: c(t)=c_{0}+1\right\} .
$$

Then (57) implies that $0<T\left(\varphi_{0}\right)<\infty$ and it is uniformly bounded independently of $\varphi_{0}$. Proposition 17 also implies that the set $\left\{\psi:\|\psi\|_{1, b} \leqslant M / 4 D\right\}$ is mapped into itself by the nonlinear map

$$
\mathcal{S}: \varphi_{0} \mapsto \varphi\left(T\left(\varphi_{0}\right)\right) .
$$

Parabolic regularity (67) implies that it is a continuous map.

Now consider the Banach space $\mathcal{B C}$ of bounded continuous functions of $z \in \mathbb{R}$ with the uniform norm. Since the set $\left\{\psi:\|\psi\|_{1, b} \leqslant M / 4 D\right\}$ is a compact convex subset of $\mathcal{B C}$, [13, Corollary 11.2] gives the desired existence of a fixed point of $\mathcal{S}$.

\section{Analytical examples}

This section provides two analytical examples of the pinning and de-pinning behaviour, one in the semilinear graph case and the other in the reaction-diffusion case. 


\subsection{Semilinear graph case}

Consider for $\delta>0$ the equation

$$
0=u_{x x}+\delta \sin (2 \pi u(x)) \cos (2 \pi x)+F, \quad u(0)=u(1) .
$$

We have the following lemma:

Lemma 20 . Let $0<\delta<1 / 4 \pi$ so that $2 \pi \delta /(1-2 \pi \delta)<1$. Then for $F>\delta^{2} 2 \pi /(1-2 \pi \delta)$, (59) does not have a solution.

2. Let $0<\delta<1 / 2$. Then for $0<F<\delta^{2}\left(1 / 4-2 \delta^{3}\right)$, 59 has a stable stationary solution.

REMARK. Statement 2 shows that there is pinning while statement 1 shows that the de-pinning is nontrivial, i.e. it occurs before $\delta \sin (2 \pi u(x)) \cos (2 \pi x)+F$ has a definite sign. Note that the ranges of the parameters are not expected to be sharp for $\delta$ small.

Proof. For statement 1 , the claim is obvious if $F>\delta$. Hence assume $F=f \delta, 0<f<1$. We argue by contradiction and assume that there exists a stationary solution $u$. By standard regularity this solution is smooth, and because it is periodic, there exists an $x_{0} \in[0,1]$ such that $u^{\prime}\left(x_{0}\right)=0$. Hence $\left|u^{\prime}(x)\right| \leqslant \delta(1+f)$ and $|u(x)-u(0)| \leqslant \delta(1+f)$.

Integrating [59] over $[0,1]$ we obtain

$$
\begin{aligned}
0= & \delta f+\delta \int_{0}^{1} \sin (2 \pi u(0)) \cos (2 \pi x) \mathrm{d} x \\
& +\delta \int_{0}^{1}\left(\cos (2 \pi x)(u(0)-u(x)) 2 \pi \int_{0}^{1} \cos (2 \pi\{(1-s) u(0)+s u(x)\}) \mathrm{d} s\right) \mathrm{d} x \\
\geqslant & \delta f-2 \pi \delta \sup _{0<x<1}|u(0)-u(x)| \geqslant \delta(f-2 \pi \delta(1+f))>0,
\end{aligned}
$$

which proves the first claim.

For statement 2, we will construct sub- and supersolutions. The comparison principle implies that there exists a stable solution between the sub- and the supersolution. We write $F=\delta^{2} f$, $0<f<1$. Let $\rho>\delta^{3}$ and choose $c^{ \pm} \in(1 / 8,3 / 8)$ such that

$$
\cos \left(2 \pi c^{ \pm}\right) \sin \left(2 \pi c^{ \pm}\right)=-4 \pi(f \mp \rho)
$$

which is possible for $f \leqslant(8 \pi)^{-1}-\delta^{3}$. Let

$$
u^{ \pm}(x):=c^{ \pm}+\delta(2 \pi)^{-2} \sin \left(2 \pi c^{ \pm}\right) \cos (2 \pi x)-\frac{1}{2}(2 \pi)^{-2} \delta^{2}(f \mp \rho) \cos ^{2}(2 \pi x) .
$$

Then

$$
\begin{aligned}
u_{x x}^{ \pm}= & -\delta \sin \left(2 \pi c^{ \pm}\right) \cos (2 \pi x)+\delta^{2} 2(f \mp \rho) \cos ^{2}(2 \pi x)-\delta^{2}(f \mp \rho), \\
\delta \sin \left(2 \pi u^{ \pm}\right) \cos (2 \pi x)= & \delta \sin \left(2 \pi c^{ \pm}\right) \cos (2 \pi x)+\delta^{2}(2 \pi)^{-1} \cos \left(2 \pi c^{ \pm}\right) \sin \left(2 \pi c^{ \pm}\right) \cos ^{2}(2 \pi x) \\
& -\frac{\delta^{3}}{2}(2 \pi)^{-1} \cos \left(2 \pi c^{ \pm}\right)(f \mp \rho) \cos ^{3}(2 \pi x)+O\left(\delta^{3}\right) .
\end{aligned}
$$

Hence $u_{x x}^{ \pm}+\delta \sin \left(2 \pi u^{ \pm}\right) \cos (2 \pi x)+\delta^{2} f= \pm \delta^{2} \rho+O\left(\delta^{3}\right)$ so that

$$
\begin{aligned}
& u_{x x}^{+}+\delta \sin \left(2 \pi u^{+}\right) \cos (2 \pi x)+\delta^{2} f \geqslant \delta^{2} \rho-O\left(\delta^{3}\right)>0, \\
& u_{x x}^{-}+\delta \sin \left(2 \pi u^{-}\right) \cos (2 \pi x)+\delta^{2} f \leqslant-\delta^{2} \rho+O\left(\delta^{3}\right)<0,
\end{aligned}
$$

i.e. the desired sub- and supersolutions exist. 


\subsection{Reaction-diffusion (Allen-Cahn) case}

This example is not constrained by the heterogeneity being weak and yet the exact values of the threshold force and the relevant constant in the time asymptotic (9) can be found. The computations are formal but yet illustrative. Consider the equation

$$
v_{t}=v_{x x}-\frac{W^{\prime}(v)}{2}+A \sin \left(\frac{x}{L}\right)+F
$$

where $0<A, F \ll 1$ and $W(\cdot)$ is chosen to be the the bilinear double-well potential

$$
W(p)=(|p|-1)^{2} \quad \text { so that } \quad W^{\prime}(p)= \begin{cases}2(p-1) & \text { for } p>0, \\ 2(p+1) & \text { for } p<0 .\end{cases}
$$

We first look for stationary solutions of (60) with one transitional interface-there is one single value of $z$ at which $v$ changes its sign. Under this assumption, the solution $v$ takes the form

$$
\begin{aligned}
v_{x x}-v+1+A \sin \left(\frac{x}{L}\right)+F & =0 \quad \text { for } x \leqslant z \\
v_{x x}-v-1+A \sin \left(\frac{x}{L}\right)+F & =0 \quad \text { for } x \geqslant z \\
v\left(z^{-}\right), v\left(z^{+}\right) & =0 \\
v_{x}\left(z^{-}\right) & =v_{x}\left(z^{+}\right) .
\end{aligned}
$$

In the above, we implicitly assume that $v>(<) 0$ for $x<(>) z$. Now $v$ is explicitly given by

$$
v(x)= \begin{cases}-e^{x-z}+\frac{L^{2}}{1+L^{2}} A \sin \left(\frac{x}{L}\right)+F+1 & \text { for } x<z, \\ e^{-(x-z)}+\frac{L^{2}}{1+L^{2}} A \sin \left(\frac{x}{L}\right)+F-1 & \text { for } x>z .\end{cases}
$$

We also have the following expressions for the value of $z$ and the slope of $v$ at $z$ :

$$
\begin{aligned}
\sin \left(\frac{z}{L}\right) & =-\frac{F}{A} \frac{1+L^{2}}{L^{2}} \\
v_{x}(z) & =-1+\frac{A L}{1+L^{2}} \cos \left(\frac{z}{L}\right) .
\end{aligned}
$$

From 61 , we see that the threshold force is given by

$$
F_{*}=\frac{A L^{2}}{1+L^{2}}<1,
$$

so that at the threshold, the spatial inhomogeneity still changes sign so that de-pinning is also nontrivial.

The above value of $F_{*}$ coincides with the forcing which makes the stationary solution just lose its stability. This is illustrated as follows. The eigenvalue problem of the linearised operator for the stationary solution of 60 is written formally as

$$
\varphi_{x x}-\frac{W^{\prime \prime}(v)}{2} \varphi=\lambda \varphi
$$


If we assume without loss of generality that $z=0$, the above problem takes the form

$$
\begin{aligned}
\varphi_{x x}-\varphi & =\lambda \varphi \quad \text { for } x \neq 0, \\
\varphi_{x}\left(0^{+}\right)-\varphi_{x}\left(0^{-}\right) & =-\frac{2 \varphi(0)}{\left|v_{x}(0)\right|}, \\
\varphi\left(0^{+}\right) & =\varphi\left(0^{-}\right) .
\end{aligned}
$$

The principal eigenfunction $\varphi_{p}(x)$ (which is positive) and eigenvalue $\lambda_{p}$ are given by

$$
\varphi_{p}(x)=\left\{\begin{array}{ll}
e^{\sqrt{1+\lambda_{p}} x}, & x \leqslant 0, \\
e^{-\sqrt{1+\lambda_{p}} x}, & x \geqslant 0,
\end{array} \quad \lambda_{p}=\frac{1}{\left|v_{x}(0)\right|^{2}}-1 .\right.
$$

Hence $v$ is stable, i.e. $\lambda_{p}<0$ if and only if $\left|v_{x}(0)\right|>1$.

Next we will compute the constant $A$ of 9 .

\section{A. Short time regularity estimates}

This section provides some finite time regularity estimates for semilinear parabolic differential equations. The results are crude, but useful as an initial step to obtain more refined estimates. Here we only give the properties relevant to this paper, in particular in the weighted spaces $\mathcal{B}_{1, b}$ from 48$]$.

Proposition 21 Consider the equation

$$
u_{t}=u_{x x}+f\left(u, u_{x}\right)+g(x, t), \quad u(x, 0)=u_{0}(x) .
$$

Assume $f$ is a $C^{1}$ function satisfying $|f(p, q)| \leqslant C(|p|+|q|)$, $\sup _{p, q}\left\{\left|f_{p}\right|,\left|f_{q}\right|\right\}<\infty$ and $\|g(\cdot, t)\|_{0, b}<\infty$ for all $t$. Then for all $T>0$, there exists a $C(T)$ such that

$$
\begin{aligned}
& \|u(\cdot, t)\|_{0, b} \leqslant C(T)\left\{\left\|u_{0}\right\|_{0, b}+\sup _{t \in[0, T]}\|g(\cdot, t)\|_{0, b}\right\}, \\
& \|u(\cdot, t)\|_{1, b} \leqslant C(T)\left\{\left\|u_{0}\right\|_{0, b} / \sqrt{t}+\sup _{t \in[0, T]}\|g(\cdot, t)\|_{0, b}\right\}, \\
& \|u(\cdot, t)\|_{1, b} \leqslant C(T)\left\{\left\|u_{0}\right\|_{1, b}+\sup _{t \in[0, T]}\|g(\cdot, t)\|_{0, b}\right\} .
\end{aligned}
$$

If further, $\|g(\cdot, t)\|_{1, b}<\infty$, then

$$
\left\|u_{x}(\cdot, t)\right\|_{1, b} \leqslant C(T)\left\{\left\|u_{0}\right\|_{1, b} / \sqrt{t}+\sup _{t \in[0, T]}\|g(\cdot, t)\|_{1, b}\right\} .
$$

REMARK. The proof is omitted as the technique of proof can be found in many standard texts such as [12, 19]. But we remark that since $f$ is globally Lipschitz, the results can be obtained by analysing the linear equation

$$
u_{t}=u_{x x}+A(x, t) u+B(x, t) u_{x}+C(x, t)
$$

with $L^{\infty}$ coefficients. Standard iteration steps then produce the estimates (64)-67) for the fully nonlinear equation (63).

The purpose of considering (63) is to analyse (44) which originates from (2). As the nonlinearity $W^{\prime}(\cdot)$ satisfies $u W^{\prime}(u) \geqslant c u^{2}$ for $|u|$ large enough, it follows that with the $L^{\infty}$ source term $\delta(g(x)+F)$, the space $\left\{\|u\|_{\infty}<C\right\}$ is invariant under the flow of $(2)$. In this setting, we can assume without loss of generality that the nonlinear term is globally Lipschitz. 


\section{B. Spectral analysis of the Allen-Cahn operator}

This section analyses the spectral and decay properties for the linear equation given by $L_{W}$ (see (54)). The approach adopted here is based on [24]. For simplicity, we will suppress the subscript $W$.

Let $V_{+}=-W^{\prime \prime}(1), V_{-}=-W^{\prime \prime}(-1)$, and $V_{*}=\max V_{ \pm}$. For a given complex number $\lambda$, consider the linear equation

$$
(L-\lambda) \varphi=0 .
$$

The parameter $\lambda$ will be taken from

$$
\Omega_{\alpha, \beta}=\left\{\left|\arg \left(\lambda-V_{*}\right)\right|<\pi-\alpha\right\} \cap\left\{\left|\lambda-V_{*}\right|>\beta\right\} \quad \text { for some } 0<\alpha<\pi / 2 \text { and } \beta>0 .
$$

The expressions $\gamma_{ \pm}(\lambda)=\sqrt{\lambda-V_{ \pm}}$appear often in the analysis. They are taken to be single-valued and analytic in $\Omega_{0,0}$.

Equation (68) has solutions $\varphi_{i}(\lambda, x)$ and $\psi_{i}(\lambda, x)(i=1,2)$ such that $\Omega_{0,0} \ni \lambda \rightarrow \infty$; they satisfy (the prime' refers to the $x$-derivative):

$$
\begin{gathered}
\text { for } x \geqslant 0:\left\{\begin{array}{l}
\varphi_{1}(\lambda, x)=e^{-\gamma_{+}(\lambda) x}\left(1+O\left(|\lambda|^{-1 / 2}\right)\right), \\
\varphi_{1}^{\prime}(\lambda, x)=e^{-\gamma_{+}(\lambda) x}\left(-\gamma_{+}(\lambda)+O(1)\right), \\
\varphi_{2}(\lambda, x)=e^{\gamma_{+}(\lambda) x}\left(1+O\left(|\lambda|^{-1 / 2}\right)\right), \\
\varphi_{2}^{\prime}(\lambda, x)=e^{\gamma_{+}(\lambda) x}\left(\gamma_{+}(\lambda)+O(1)\right),
\end{array}\right. \\
\text { for } x \leqslant 0:\left\{\begin{array}{l}
\psi_{1}(\lambda, x)=e^{\gamma_{-}(\lambda) x}\left(1+O\left(|\lambda|^{-1 / 2}\right)\right), \\
\psi_{1}^{\prime}(\lambda, x)=e^{\gamma_{-}(\lambda) x}\left(\gamma_{-}(\lambda)+O(1)\right), \\
\psi_{2}(\lambda, x)=e^{-\gamma_{-}(\lambda) x}\left(1+O\left(|\lambda|^{-1 / 2}\right)\right), \\
\psi_{2}^{\prime}(\lambda, x)=e^{-\gamma_{-}(\lambda) x}\left(-\gamma_{-}(\lambda)+O(1)\right),
\end{array}\right.
\end{gathered}
$$

In addition, the functions $\varphi_{i}$ and $\psi_{i}$ can be related in the following way:

$$
\begin{aligned}
\varphi_{i}(\lambda, x) & =A_{i}(\lambda) \psi_{1}(\lambda, x)+B_{i}(\lambda) \psi_{2}(\lambda, x), \\
\psi_{i}(\lambda, x) & =C_{i}(\lambda) \varphi_{1}(\lambda, x)+D_{i}(\lambda) \varphi_{2}(\lambda, x),
\end{aligned}
$$

where the coefficients $A_{i}, B_{i}, C_{i}$ and $D_{i}$ are analytic functions of $\lambda \in \Omega_{0,0}$ and can all be shown to be uniformly bounded as $\Omega_{0,0} \ni \lambda \rightarrow \infty$.

Recall the definitions (47) and (48) of the weighted function spaces $\mathcal{B}_{0, b}$ and $\mathcal{B}_{1, b}$. We have the following main estimates in these spaces.

Lemma 22 Given $0<\alpha<\pi / 2, \beta>0$ and the domain $\Omega_{\alpha, \beta}$, let

$$
0<b<\frac{1}{2} \min \left\{\Re \gamma_{+}(\lambda), \Re \gamma_{-}(\lambda): \lambda \in \Omega_{\alpha, \beta}\right\} .
$$

Then the following two statements hold.

(I) For any $\lambda \in \Omega_{\alpha, \beta}$, if the Wronskian

$$
W\left(\varphi_{1}, \psi_{1}, \lambda\right)=\varphi_{1}(x, \lambda) \psi_{1}^{\prime}(x, \lambda)-\varphi_{1}^{\prime}(x, \lambda) \psi_{1}(x, \lambda) \neq 0,
$$

then the resolvent operator $(L-\lambda)^{-1}$ exists as a bounded operator on $\mathcal{B}_{1, b}$. 
(II) There exists a constant $C(\alpha, \beta)$ such that for any large enough $\lambda \in \Omega_{\alpha, \beta}$, the resolvent operator $(L-\lambda)^{-1}$ exists and the following estimates hold:

$$
\begin{aligned}
& \left\|(L-\lambda)^{-1} g\right\|_{0, b} \leqslant \frac{C(\alpha, \beta)}{|\lambda|}\|g\|_{0, b}, \\
& \left\|(L-\lambda)^{-1} g\right\|_{1, b} \leqslant \frac{C(\alpha, \beta)}{\sqrt{|\lambda|}}\|g\|_{0, b}, \\
& \left\|(L-\lambda)^{-1} g\right\|_{1, b} \leqslant \frac{C(\alpha, \beta)}{|\lambda|}\|g\|_{1, b} .
\end{aligned}
$$

Proof. The proof relies on estimating the Green's function of the resolvent:

$$
G(x, y, \lambda)=\frac{1}{W\left(\varphi_{1}, \psi_{1}, \lambda\right)} \begin{cases}\psi_{1}(y, \lambda) \varphi_{1}(x, \lambda), & x>y \\ \varphi_{1}(y, \lambda) \psi_{1}(x, \lambda), & x<y .\end{cases}
$$

Note that $W$ is independent of $x$. Hence $G(x, y, \lambda)$ is well defined if $W \neq 0$. We will only prove statement (II) 75, 76, 77), statement (I) follows immediately.

Using the estimates for $\varphi$ and $\psi$, it is possible to show that as $\lambda \rightarrow \infty$ :

$$
W\left(\varphi_{1}, \psi_{1}, \lambda\right)=-\left(\gamma_{+}(\lambda)+\gamma_{-}(\lambda)\right)+O(1)=O(\sqrt{\lambda})
$$

Hence if $\lambda \in \Omega_{\alpha, \beta}$ and is large enough, $W(\lambda)$ does not vanish and thus $G$ is well defined. In this case, the function $f=(L-\lambda)^{-1} g$ can be written as

$$
\begin{aligned}
f(x) & =\int_{-\infty}^{\infty} G(x, y, \lambda) g(y) \mathrm{d} y \\
& =\frac{1}{W(\lambda)}\left\{\int_{-\infty}^{x} \varphi_{1}(x, \lambda) \psi_{1}(y, \lambda) g(y) \mathrm{d} y+\int_{x}^{\infty} \psi_{1}(x, \lambda) \varphi_{1}(y, \lambda) g(y) \mathrm{d} y\right\} .
\end{aligned}
$$

Suppose $\|g\|_{1, b}<\infty$. We will estimate $\|f\|_{0, b}$ as follows. Let $x>0$. The first term of (78) is bounded by

$$
\begin{aligned}
& \left|\frac{\varphi_{1}(x, \lambda)}{W(\lambda)}\right|\left\{\int_{-\infty}^{0}\left|\psi_{1}(y, \lambda) g(y) e^{-b y} e^{b y}\right| \mathrm{d} y+\int_{0}^{x}\left|\psi_{1}(y, \lambda) g(y) e^{b y} e^{-b y}\right| \mathrm{d} y\right\} \\
& \quad \leqslant\left|\frac{\varphi_{1}(x, \lambda)}{W(\lambda)}\right|\left\{\int_{-\infty}^{0}\left|\psi_{1}(y, \lambda) e^{b y}\right| \mathrm{d} y+\int_{0}^{x}\left|\psi_{1}(y, \lambda) e^{-b y}\right| \mathrm{d} y\right\}\|g\|_{0, b} \\
& \quad \leqslant C\left|\frac{e^{-\Re \gamma_{+}(\lambda) x}}{W(\lambda)}\right|\left\{\int_{-\infty}^{0} e^{\Re \gamma_{-}(\lambda) y} e^{b y} \mathrm{~d} y+\int_{0}^{x} D_{1}(\lambda) e^{\Re \gamma_{+}(\lambda) y} e^{-b y} \mathrm{~d} y\right\}\|g\|_{0, b} \quad \text { (by (73)) } \\
& \leqslant C\left|\frac{e^{-\Re \gamma_{+}(\lambda) x}}{W(\lambda)}\right|\left\{\frac{1}{\Re \gamma_{-}(\lambda)+b}+\frac{e^{\left(\Re \gamma_{+}(\lambda)-b\right) x}-1}{\Re \gamma_{+}(\lambda)-b}\right\}\|g\|_{0, b} \leqslant \frac{C e^{-b x}}{|\lambda|}\|g\|_{0, b}
\end{aligned}
$$

where we have used the fact that $D_{1}(\lambda)$ is bounded. The second term of $(78)$ is estimated as

$$
\begin{aligned}
\left|\frac{\psi_{1}(x, \lambda)}{W(\lambda)}\right| \int_{x}^{\infty}\left|\varphi_{1}(y, \lambda) g(y) e^{b y} e^{-b y}\right| \mathrm{d} y & \leqslant C\left|\frac{e^{\Re \gamma_{+}(\lambda) x}}{W(\lambda)}\right| \int_{x}^{\infty} e^{-\left(\Re \gamma_{+}(\lambda)+b\right) y} \mathrm{~d} y\|g\|_{1, b} \\
& \leqslant \frac{C e^{-b x}}{|\lambda|}\|g\|_{0, b} .
\end{aligned}
$$

Similar estimates can be obtained for $x<0$. Thus $(75)$ is proved. 
Estimate 76 is proved in exactly the same way. Taking the derivative brings down a $\sqrt{|\lambda|}$. Estimate (77) is proved by the following observation:

$$
\left(f_{x}\right)_{x x}+(V(x)+\lambda) f_{x}=g_{x}+V^{\prime}(x) f,
$$

i.e.

$$
\left\|f_{x}\right\|_{0, b} \leqslant \frac{C\|g\|_{1, b}}{|\lambda|}+\|f\|_{0, b} \leqslant \frac{C\|g\|_{1, b}}{|\lambda|}+\frac{C\|g\|_{0, b}}{|\lambda|} \leqslant \frac{C\|g\|_{1, b}}{|\lambda|} .
$$

Next we introduce the concept of eigenvalues of $L$.

Definition 23 A complex number $\lambda$ is called an eigenvalue of $L$ if there exists a bounded function $\varphi \not \equiv 0$ such that $(L-\lambda) \varphi=0$.

A complex number $\lambda$ is said to belong to the resolvent set of $L$ if $(L-\lambda)^{-1}$ exists as a bounded operator on the space of bounded functions, i.e. $\mathcal{B}_{0,0}$.

With the above, we have the following lemma (which basically summarises the results of [24, Lemma 5.3 and Theorem 5.5]).

Lemma 24 1. The eigenvalues of $L$ are real. Furthermore, those eigenvalues in the interval $\left(V_{*}, \infty\right)$ are discrete and actually confined in $\left(V_{*},\|V\|_{\infty}\right)$ and can only cluster at $V_{*}$.

2. Since $m^{\prime}(x)>0$ is an eigenfunction with respect to $\lambda=0$, the operator $L$ has no positive eigenvalues. The previous statement then implies that there is a spectral gap, i.e. there is a positive distance between the principal eigenvalue, 0 , and the the second largest eigenvalue.

3. There are $0<\alpha<\pi / 2$ and $\beta>0$ such that $(L-\lambda)^{-1}$ exists for all $\lambda \in \Omega_{\alpha, \beta}$. Hence there exist $C(\alpha, \beta)$ and $b>0$ such that the estimates (75), (76) and (77) hold for all $\lambda \in \Omega_{\alpha, \beta}$.

We now state and prove a linear decay estimate for the semigroup of (54).

THEOREM 25 Let $v(x, t)$ be the solution of

$$
v_{t}=v_{x x}-W^{\prime \prime}(m(x)) v, \quad v(x, 0)=v_{0},
$$

where $v_{0} \in \mathcal{B}_{1, b}$ and $\int_{-\infty}^{\infty} v_{0}(x) m^{\prime}(x) \mathrm{d} x=0$. Then there exist $D \geqslant 1$ and $\omega>0$ such that for all $t>0$,

$$
\begin{gathered}
\|v(t)\|_{1, b} \leqslant D \frac{e^{-\omega t}}{\sqrt{t}}\left\|v_{0}\right\|_{0, b}, \\
\|v(t)\|_{1, b} \leqslant D e^{-\omega t}\left\|v_{0}\right\|_{1, b} .
\end{gathered}
$$

Proof. From Lemma 24 3 , we can find $\omega>0$ and $\pi / 2<\gamma<\pi$ such that the spectrum of $L$ minus zero lies completely to the left of the complex curve

$$
C_{\omega, \gamma}=\left\{\lambda=-\omega+\rho e^{ \pm i \gamma}: 0<\rho<\infty\right\} .
$$

Hence the solution $v(t)$ can be written as the following contour integral:

$$
v(t)=\frac{1}{2 \pi i} \int_{C_{\omega, \gamma}} e^{t \lambda}(\lambda-L)^{-1} v_{0} \mathrm{~d} \lambda .
$$

Choose $b$ with

$$
0<b<\frac{1}{2} \min \left\{\Re \gamma_{+}(\lambda), \Re \gamma_{-}(\lambda): \lambda \in C_{\omega, \gamma}\right\}
$$


We now estimate the solution $v(t)$ as follows:

$$
\|v(t)\|_{1, b} \leqslant \frac{1}{2 \pi} \int_{C_{\omega, \gamma}}\left|e^{t \lambda}\right|\left\|(\lambda-L)^{-1}\right\|\left\|v_{0}\right\|_{0, b}|\mathrm{~d} \lambda| \leqslant \frac{C(\alpha, \beta)}{2 \pi}\left(\int_{C_{\omega, \gamma}} \frac{\left|e^{t \lambda}\right|}{\sqrt{|\lambda|}}|\mathrm{d} \lambda|\right)\left\|v_{0}\right\|_{0, b} .
$$

The last integral is bounded by

$$
\begin{aligned}
\int_{C_{\omega, \gamma}} \frac{\left|e^{t \lambda}\right|}{\sqrt{\lambda}}|\mathrm{d} \lambda| & \leqslant 2 \int_{0}^{\infty} \frac{e^{-\omega t} e^{t \rho \cos \gamma} \mathrm{d} \rho}{\left[(\rho \cos \gamma-\omega)^{2}+\rho^{2} \sin ^{2} \gamma\right]^{1 / 4}} \leqslant 2 \int_{0}^{\infty} \frac{e^{-\omega t} e^{t \rho \cos \gamma} \mathrm{d} \rho}{\left[\omega^{2}+\rho^{2} \sin ^{2} \gamma\right]^{1 / 4}} \\
& \leqslant D e^{-\omega t} \int_{0}^{\infty} \frac{e^{t \rho \cos \gamma} \mathrm{d} \rho}{\sqrt{\rho \sin \gamma}} \leqslant D \frac{e^{-\omega t}}{\sqrt{t}}
\end{aligned}
$$

leading to $(80)$.

Finally, by parabolic regularity, for any $T>0$, there exists $C(T)$ such that

$$
\|v(t)\|_{1, b} \leqslant C(T)\left\|v_{0}\right\|_{1, b} \quad \text { for } 0<t<T .
$$

Hence by choosing a larger $D$ and a smaller $\omega$ (depending on $T$ ), the conclusion 81 also follows.

\section{Acknowledgements}

The authors would like to thank Kaushik Bhattacharya for very helpful communications which led to our interest in this problem. The hospitality of the Max-Planck Institute, Leipzig, Germany is also highly appreciated.

The first author was partially supported by the Postdoc-Program of the German Academic Exchange Service (DAAD) and the Department of Mathematics of the University of Texas at Austin and acknowledges the kind hospitality of Purdue University and the University of Texas at Austin.

The second author is partially supported by the Division of Mathematical Sciences of the National Science Foundation.

\section{REFERENCES}

1. Abeyaratne, R. A., Chu, C., \& James, R. D. Kinetics of materials with wiggly energies: theory and application to the evolution of twinning microstructures in a Cu-Al-Ni shape. Philos. Mag. A 73 (1996), 457-497.

2. Allen, S. M., \& CAHn, J. A microscopic theory for antiphase boundary motion and its application to domain coarsening. Acta Metall. 27 (1979), 1085-1095.

3. Ambrosetti, A., \& Prodi, G. A Primer of Nonlinear Analysis. Cambridge Stud. Adv. Math. 34, Cambridge Univ. Press (1993). Zbl 0818.47059 MR 1336591

4. Arao ReIs, F. D., Depinning transitions in interface growth models. Brazilian J. Phys. 33 (2003), 501-513.

5. Bertotti, G. Hysteresis in Magnetism. Academic Press Ser. Electromagnetism, Academic Press, San Diego (1998).

6. Bhattacharya, K., \& Craciun, B. Homogenization of a Hamilton-Jacobi equation associated with the geometric motion of an interface. Proc. Roy. Soc. Edinburgh Sect. A 133 (2003), 773-805. Zbl 1043.35028 MR 2006202

7. Bhattacharya, K., \& CRACIUn, B. Effective motion of a curvature-sensitive interface through a heterogeneous medium. Interfaces Free Bound. 6 (2004), 151-173. Zbl 1061.35148 MR 2079601 
8. Carpio, A., \& Bonilla, L. L. Depinning transitions in discrete reaction-diffusion equations. SIAM J. Appl. Math. 63 (2003), 1056-1082. Zbl 1035.34058 MR 1969687

9. DANCER, E. N., \& Hess, P. Stability of fixed points for order-preserving discrete-time dynamical systems. J. Reine Angew. Math. 419 (1991), 125-139. Zbl 0728.58018 MR 1116922

10. FIFE, P. C., \& MCLEOD, J. B. The approach of solutions of nonlinear diffusion equations to travelling front solutions. Arch. Rat. Mech. Anal. 65 (1977), 335-361. Zbl 0361.35035 MR 0442480

11. FISHER, D. S. Collective transport in random media: from superconductors to earthquakes. Phys. Rep. 301 (1998), 113-150.

12. Friedman, A. Partial Differential Equations of Parabolic Type. Prentice-Hall (1964). Zbl 0144.34903 MR 0181836

13. Gilbarg, D., \& Trudinger, N. S. Elliptic Partial Differential Equations of Second Order. 2nd ed., Springer (1983). Zbl 0562.35001 MR 0737190

14. Grunewald, N. Barkhausen effect: a stick-slip motion in a random medium. Methods Appl. Anal., to appear.

15. Henry, D. Geometric Theory of Semilinear Parabolic Equations. Lecture Notes in Math. 840, Springer (1981). Zbl 0456.35001 MR 0610244

16. Ioffe, L.B., \& VinoKUR, V. M. Dynamics of interfaces and dislocations in disordered media. J. Phys. C: Solid State Phys. 20 (1987), 6149-6158.

17. Kardar, M. Nonequilibrium dynamics of interfaces and lines. Phys. Rep. 301 (1998), 85-112.

18. LIONS, P.-L., \& SougAnidis, P. E. Homogenization of degenerate second-order PDE in periodic and almost-periodic environments and applications. Ann. Inst. H. Poincaré Anal. Non Linéaire 22 (2005), $667-677$.

19. Lunardi, A. Analytic Semigroups and Optimal Regularity in Parabolic Problems. Birkhäuser (1995). Zbl 0816.35001 MR 1329547

20. NAmah, G., \& RoQuejoffre, J. M. Convergence to periodic fronts in a class of semilinear parabolic equations. Nonlinear Differential Equations Appl. 4 (1997), 521-536. Zbl 0887.35070 MR 1485736

21. Nattermann, T. Theory of the random field ising model. Spin Glasses and Random Fields, A. P. Young (ed.), World Sci., Singapore (1998), 277-298.

22. Rubinstein, J., Sternberg, P., \& Keller, J. B. Fast reaction, slow diffusion, and curve shortening. SIAM J. Appl. Math. 49 (1989), 116-133. Zbl 0701.35012 MR 0978829

23. Rubio, M. A., Edwards, C. A., Dougherty, A., \& Gollub, J. P. Self-affine fractal interfaces from immiscible displacement in porous media. Phys. Rev. Lett. 63 (1989), 1685-1688.

24. SAttinger, D. H. On the stability of waves of nonlinear parabolic systems. Adv. Math. 22 (1976), 312355. Zbl 0344.35051 MR 0435602

25. Souganidis, P. E. Front propagation: theory and applications. Viscosity Solutions and Applications (Montecatini Terme, 1995), Lecture Notes in Math. 1660, Springer (1997), 186-242. Zbl 0882.35016 MR 1462703

26. XIN, J. Existence and stability of travelling waves in periodic media governed by a bistable nonlinearity. J. Dynamical Differential Equations 3 (1991), 541-573. Zbl 0769.35033 MR 1129560

27. XIN, J. Existence and non-existence of travelling waves and reaction-diffusion front propagation in periodic media. J. Statist. Phys. 73 (1993), 893-926. MR 1251222

28. XIN, J., \& ZHU, J. Quenching and propagation of bistable reaction-diffusion fronts in multidimensional periodic media. Phys. D 81 (1995), 94-110. Zbl 0900.35193 MR 1313470 